\title{
Displacement-Based Assessment of Ductile Tearing under Low-Constraint Conditions
}

\author{
V.P. Naumenko*, S.V. Lenzion and I.V. Limansky \\ Department of Structural Integrity, G. S. Pisarenko Institute for Problems of Strength, Kyiv, Ukraine
}

\begin{abstract}
This paper deals with several issues in the ductile tearing assessment relevant to the fracture criteria and test methods covering the determination of the crack growth resistance under low-constraint conditions. Specifically, the effects of the specimen geometry and size, type and length of the original stress raiser, boundary restraints, load biaxiality, loading history, and plastic anisotropy were studied experimentally. With regard to the problem of transferring crack growth data, our results demonstrate that underlying assumptions of the current concept of ductile tearing evoke a suspicion from different points of view. To clarify the reasons for a gap between the model descriptions and measurements, we put forward an innovative engineering concept enabling through-life assessment of the fracture process in sheet metals.
\end{abstract}

Keywords: Plane stress fracture, crack parameters, load biaxiality, thin-sheet aluminium, steady state tearing.

\section{INTRODUCTION}

Thin-walled components find many applications in aerospace, mechanical, civil, and ocean engineering. The nucleation and propagation of crack-like flaws in such components of ductile materials is often accompanied by the development of low-constraint flow fields under the uniform distribution of tensile or compressive stresses. It is of scientific and practical interest to develop a general fracture criterion and a test method such that the nucleation and extension of a single tear crack in damage-tolerant components made from metallic, nonmetallic or composite materials could be assessed in a unified manner. This is the main reason behind the long-standing efforts of our research team towards the development of a general concept of Mode I fracture under biaxial loading in tension and/or compression.

To establish the basic principles of this concept, called the Unified Methodology (UM) of fracture investigation, we performed an extensive experimental study of stable crack growth in brittle and ductile materials [1-17]. Focus was on the fracture behaviour of a single through crack in plates and tubes subjected to monotonic loading in tension and compression. Specimens of different geometries and sizes were made from notionally homogeneous metallic and non-metallic materials with widely varying properties. The overall objective of the UM development is to formulate a Transferring Law (TL), i.e., a common function for experimental data on stable crack growth in simple specimens of a relatively small size and large-scale components of complicated geometry. This function can be seen as a key result of the coordinated research efforts towards developing an advanced, coherent, and harmonised Fitness-For-Service (FFS) procedure for a through-life assessment of tearing in thin-walled

*Address correspondence to this author at the G. S. Pisarenko Institute for Problems of Strength, 2 Tymiriazievska str., Kyiv 01014, Ukraine;

E-mail: v.p.naumenko@ipp.kiev.ua components made from sheet materials of any physical nature. Because of the breadth of this research programme, the UM concept remains at the development stage and the related exploratory studies are still in progress.

It should be emphasised that in the most practical applications of thin-walled components, the accumulation of structural damage, nucleation of a tear crack and its extension occur under biaxial loading. Presently, a well-known lack of consensus exists with respect to the magnitude and direction of the differences in the biaxial fracture behaviour as compared to the uniaxial tension. According to Eftis et al. [18] "...the theories of Griffith and Irwin are incapable of proper treatment of biaxial effect". What is more, they are also conceptually incapable to describe Mode I crack growth in brittle materials under uniform uniaxial compression, i.e., at an extremely low level of in-plane constraint.

Much literature evidence exists in support of both statements. For example, Mode I fracture was repeatedly observed in thin-walled tubes and plates of highly brittle (glassy) materials $[5,7-9,11,17]$ when they were tested under uniform compression. In these tests, through cracks were growing in a well-controlled manner under zero and negative values of the applied stress intensity factor $K_{\mathrm{I}}$. Of course, sufficient knowledge in this area of fracture mechanics is not yet available and additional research endeavours are highly needed. This is true even for the simplest case where the fracture toughness $\left(K_{\mathrm{c}}\right)$ of brittle metallic and non-metallic materials should be determined on standard specimens of the same type under tensile loading. As regards tearing in ductile materials, it is incomprehensible how the residual strength of a large-scale component with globally elastic fracture behaviour could be predicted from the data obtained on laboratory-sized specimen fractured under general yielding. This explains why the problem of plane stress fracture under biaxial loading has not yet been covered by the European FFS procedure FITNET [19].

Further development of the UM is addressed in two parts. The first one consists in continuing our long-standing search for an inherently consistent model description of biaxial 
fracture in brittle and ductile materials with notionally homogeneous mechanical properties. And the second part is related to the development of a test method for a unified through-life assessment of crack growth in damage-tolerant components operated under low-constraint conditions.

Initially, we intend to compare current fracture criteria and test methods with those used in the UM concept of plane stress tearing. It will be done through conducting an in-depth study of stable crack growth in typical sheet metals widely differing in their mechanical behaviour. The object is to develop, using a minimum number of parameters, a basic TL, which would allow predicting the residual strength of thin-walled components fractured under uniaxial and proportional biaxial loading. Due to the complexity of the problem, the subject matter of these research efforts was reduced to the greatest possible simplicity. Ideally, we intend to deal with only two identical tips of the same internal crack extending within a single straight neck.
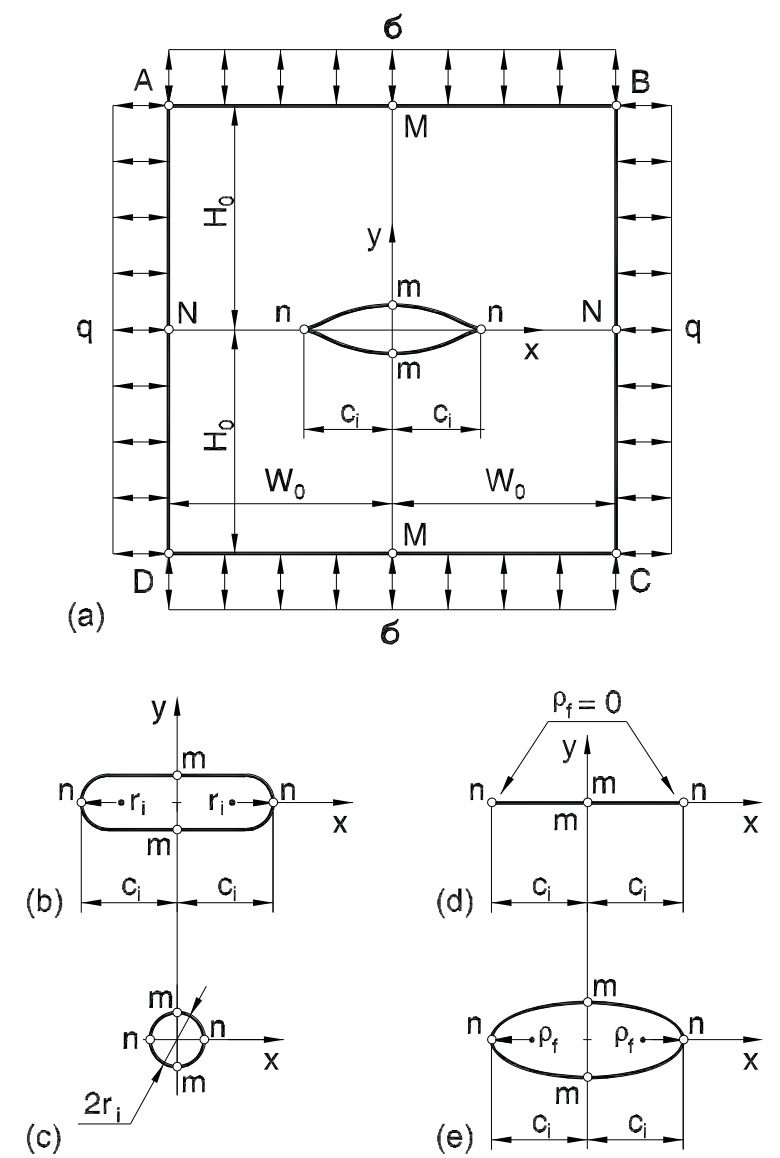

Fig. (1). The basic structural element $A B C D$ with an original geometric imperfection of length $2 c_{\mathrm{i}}$ (a) and stress raisers having a well-defined geometry of their surfaces, which are often used in fracture mechanics testing (b), (c) and analysis (d), (e).

The problem in question is confined to Steady State Tearing (SST) in a square plate shown in Fig. (1a). Both in-plane geometry and stress state of this plate, called the Basic Structural Element (BSE), are symmetric with respect to the $x$ - and $y$-axes. At the SST stage of the fracture process, the crack-tip stress-strain fields and localised necks, moving ahead the crack tips, can be considered fully developed and self-similar. The length $2 c$ of the SST crack is taken as $2 c>\kappa B_{0}$, where $B_{0}$ is the original thickness of the BSE and $\kappa$ is some quotient much larger than 1.0. Thus, the problem under investigation can be readily approximated by a two-dimensional state of generalised plane stress. When the crack tips are advancing synchronously, the outer BSE boundaries move freely under a prescribed value of the remote stress biaxiality ratio $k=q / \sigma$. With regard to the future FFS procedure, the BSE can be treated as a square element removed from a component that is subjected to specific operating conditions.

\section{Brief Outline of the State-of-the-Art}

Over the years there has been an urgency to develop valid fracture criteria and standard test methods for a unified assessment of the residual strength of thin-walled components with through cracks growing under low-constraint conditions. As to this research field, the majority of up-to-date advancements are reflected in papers of the Special Issue on Fundamentals and Applications of the CTOA [20], International Standardisation Organisation working document ISO/TC 164/SC 4 N413.3 [21], and ASTM standard E2472-06 [22]. They all underline the worldwide interest in the Crack Tip Opening Angle (CTOA- $\psi$ ) and the Crack Tip Opening Displacement (CTOD- $\delta$ ) as local fracture parameters for metallic materials. Previously, it was pointed out [23] that a single fracture parameter is sufficient to describe the crack-tip stress and strain fields under plane stress conditions. So, a wide consensus exists in the fracture mechanics community that a one-parameter characterisation of stable crack growth under uncontained yielding is conceptually possible and resistance to tearing, at least in sheet metals, can be quantified solely by a constant value $\psi_{\mathrm{c}}$ of the CTOA- $\psi$ parameter.

In order to complete the existing flaw assessment procedure SINTAP, the CTOD- $\delta_{5}$ parameter was proposed $[24,25]$ as a thin-wall option of the SINTAP. Thus, the general CTOD term should be replaced with the CTOD- $\delta_{5}$ anticipating an implicit use of the stress intensity factor $K$ and $J$-integral in the related FFS procedure. This way towards the development of a general engineering concept that would cover the whole range of industrial applications is not by far the best. There are an abundance of papers reflecting a longstanding controversy whether the R-curves expressed in terms of the $K, J, \mathrm{CTOA}-\psi$ and $\mathrm{CTOD}-\delta$, i.e., the local fracture parameters, are proper measures of the resistance to slow stable crack growth. A computational analysis of ductile crack growth [26] demonstrates convincingly that "no approach can be based on a single parameter resistance curve". Decisive experimental evidences of the validity of these statements are widely encountered in the literature. For instance, they are partly presented in our works [1-17, 27-29]. Taken together with a large body of related literature data, they support a common knowledge that the concepts of $K$ - and $J$-controlled crack growth in thin-sheet metals are in most cases invalid.

Although fracture toughness testing and analysis are performed by numerous research organisations, there are no standardised methods for the through-life assessment of tearing in sheet materials. Here the term through-life assessment means that all measures of fracture resistance are determined continuously from the instant of nucleation of a Naturally Forming Crack (NFC) and up to the complete loss of 
load carrying capacity. This technique of collecting and analysing test data was first used in our tests of thin plates made from a ductile metallic material. A set of the relevant experimental results, treated as the displacement-based fracture parameters, are presented in what follows. The remaining test data and analyses will be considered in two forthcoming papers entitled: "Energy-Based Assessment of Ductile Tearing under Low-Constraint Conditions" and "Comprehensive Assessment of Ductile Tearing under Low-Constraint Conditions". The latter paper will combine the recent achievements in the field and in this way will foster overcoming a number of problems that prevent completion of the FFS procedure FITNET [19].

The contribution of these studies to advancing the stateof-the-art will concern identifying: (i) where current fracture criteria and test procedures are inadequate, (ii) how they could be modified to improve the transferability of data obtained under uncontained yielding and, finally, (iii) whether or not the local measure of fracture resistance $\left(\mathrm{CTOA}-\psi_{\mathrm{c}}\right)$ can be coupled with the local and global measures of SST resistance used in the UM concept.

\section{Original Stress Raisers}

Any study on plane stress tearing in specimens or components made from thin sheets of ductile metallic materials deals with the interplay of elasticity, plasticity, necking, damage, and cracking. Generally, the life cycle of unnotched specimens tested under uniaxial or biaxial loading may be considered to comprise the following stages: (i) elastic behaviour with the initiation of plastic behaviour, (ii) diffused necking, (iii) localised necking, (iv) nucleation of an NFC and, finally, (v) its propagation up to the complete loss of load carrying capacity. For the conventional fracture mechanics analysis, the first three stages, being the precursor of the last two, usually are not related directly to the cracking behaviour. This undesirable gap impedes both improvement of fracture mechanics analyses and development of an effective FFS procedure.

In the UM, localised necking is treated as an integral part of ductile tearing both at the instant of NFC nucleation and during the whole process of crack extension. The exact location of the fracture initiation site is an ill-defined function of inevitable imperfections in the specimen geometry, loading conditions, and boundary restraints imposed by the grips, together with the variability of the material properties. To trigger the progressive process of single-site necking followed by single-site cracking in a predetermined location and direction, a variety of imperfections are employed in plane-stress fracture studies. According to the standard test methods, the specimen should contain initial fatigue precracks at the tips of the starting slot $[21,22]$. However, it is common knowledge that the crack extension resistance of metallic materials may be influenced significantly by the preloading history. In particular, a strong influence of the fatigue crack growth history on the fracture toughness of thin-sheet aluminium alloy 2024-T3 was reported in [30] for a specific value of the ratio $B_{0} / c$.

At present, there is no possibility to establish a one-toone correspondence between the initial fatigue damages near the crack tips in different specimens whose geometry and boundary restraints vary over wide ranges. That is why in the specimen preparation, special care must be taken to prevent the introduction of uncontrollable initial damages and residual stresses into the material to be tested. The UM tests are carried out on specimens with original imperfections having relatively simple geometry and a well-defined form of their tips. By convention, the specified starting slots (Figs. 1b and 1c) are taken as damage-free defects. Their dimensions 2 $c_{\mathrm{i}}$ and $2 r_{\mathrm{i}}$ are sufficiently small. It means that at the instant of fracture initiation the tensile stress $\sigma$ or compressive stress $q$ averaged across the BSE ligament depends only slightly on the variation of the notch size. At the same time, the imperfection should be sufficiently large to concentrate all thinning, hardening, plastic dissipation, and structural damage inside a single localised neck. Cracks initiated from such imperfections often raise the most concern when the residual strength of damage-tolerant components need to be assessed.

Generally, necks and cracks initiate inboard of an internal geometric imperfection with smooth surfaces [31], i.e., away from the points $n$ in Figs. (1b) and (1c). This is due to the absence of lateral stresses on the traction-free surface of the imperfection, which reduces the hydrostatic component of the local stress field and also the normal stress. As compared to the tests of specimens with a fatigue precrack, the proper amount of slow stable crack extension in a notched specimen that correlates with the initial NFC state can be established in a relatively simple and reproducible manner. This has an important bearing on the practical implementation of the UM procedure for assessing ductile tearing in thin-sheet metals. Earlier we demonstrated by experimental examples [32, 33] that an open circular hole of a specific size is the preferable geometric imperfection to avoid the complicated analysis of the transition in fracture behaviour from the "flat" to "slant" mechanism of crack extension. In specimens with this stress raiser, both nucleation and extension of the NFC occur by shear localisation, i.e. by mixed Mode I and Mode III cracking in the plane inclined at $45^{\circ}$ to the loading plane.

\section{Through-Life Assessment of Plane Stress Tearing}

The UM test method should provide an easy-to-use tool for through-life assessment of crack growth in laboratory-sized specimens showing purely elastic, elastic-plastic, and purely plastic fracture behaviour. For an engineering concept of such a wide scope, it is appropriate to operate with mechanical parameters that can be quantified using simple and welldefined procedures. Therefore, the TL we are searching for should be based mostly on the analysis of data that are collected by macroscopic measurements in the form of test records loads vs. displacements vs. crack extensions. Here, a special attention is given to the displacements of the so-called extreme points $m, n$ on the inner and $M, N$ on the outer BSE boundaries (Fig. 1a). The displacements $v(\mathrm{~m}), u(\mathrm{n})$, taken together with the displacements $v(\mathrm{M}), u(\mathrm{~N})$, serve as the main geometric variables needed for linking changes in the geometry of a growing crack with those of the outer boundaries. When developing the UM, we realised from the outset that no parameter of near-crack-tip fields and no micromechanically-based description of the fracture process can be incorporated into a sufficiently general and practical computational procedure unless the above linkage is properly described. 


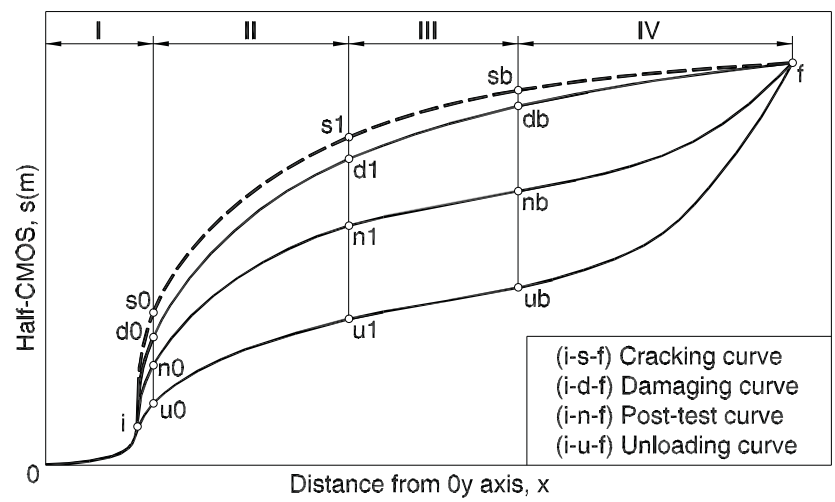

Fig. (2). Schematic presentation of through-life fracture curves relating to each other by imaginary (instantaneous) unloading reloading cycles made at the moment " 0 " of the NFC nucleation (states $\mathrm{s} 0-\mathrm{u} 0-\mathrm{d} 0$ ), moment " 1 " of the onset of SST crack growth (states $\mathrm{s} 1-\mathrm{u} 1-\mathrm{d} 1$ ), and moment " $\mathrm{b}$ " of the transition to the TET stage of fracture process (states sb-ub-db).

The UM focuses on changes in the geometry of the whole crack border, instead of considering mainly the crack-tip displacements, which are given much attention in current fracture mechanics analyses. Ductile tearing is seen as an interplay of four concurrent processes represented by the through-life fracture curves (Fig. 2), which characterise the step-wise crack growth ( $i-s-f$ curve), accumulation of localised damage ahead of the crack tips until the critical level is reached $(i-d-f)$, attainment of the zero value of stresses in the immediate vicinity of the crack tips $(i-n-f)$, and formation of specific stress-strain fields in the fullyunloaded specimen $(i-u-f)$. These curves, excluding $(i-n-f)$, can be readily plotted using the test records $P$ vs. $2 c$ and $P$ vs. $2 s(\mathrm{~m})$, where $2 s(\mathrm{~m})$ is the Crack Mouth Opening Spacing (CMOS) measured between points $m$ (Fig. 1).

To construct the post-test fracture curve $(i-n-f)$, the crack border distance $2 s(\mathrm{~m})_{\mathrm{n}}$ and the corresponding crack length $2 c$ should be measured in pairs using the upper and lower halves of the fully fractured specimen. A quarter of the upper crack border is sketched in Fig. (3a). The virtual crack extension is modelled by moving the upper specimen half towards its lower counterpart, as it was described by Lloyd [34] for an idealised fracture of a middle cracked specimen. Thus, the changes in the geometric parameters of the inner and outer boundaries should be assessed jointly for a loaded (moving crack), fully unloaded (arrested crack), and broken-down (fully developed crack) specimen.

Assume that an undeformed undamaged unstressed specimen of the BSE type contains a small stress raiser with the simplest geometry (Fig. 1c). Shortly after the application of monotone tensile load $\sigma$, two localised necks start forming in the vicinity of the extreme points $n$. The necks concentrate plastic deformation and damage inside Active Damage Zones (ADZ). Each zone encompasses some volume of severely transformed material with specific damage morphology. The structural damage accumulated inside the ADZs reaches its critical level at the instant $i$ (stage I in Fig. 2). During further loading, both zones simultaneously become fully developed just before the moment where an NFC starts to grow (state $s 0$ in Fig. 2). The NFC comprises two tear cracks that freely nucleate inboard of the plate and then come to the surfaces of the original stress raiser by the mechanism of internal necking near the points $n$ (Fig. 1c).

Immediately after the nucleation, the NFC starts to propagate with an intermittent attainment of the local instabilities in a step-wise manner (stage II in Fig. 2). The repeated cycles loading-partial unloading-reloading generate a cyclic variation in the crack profile geometry, which is bounded by the cracking and damaging curves shown in Fig. (2). Thus, tearing is thought of as the incubation of localised damage followed by the material separation in the ADZs. The SST regime of crack extension is attained at the instant $s 1$ (stage III), when the alternating process of cracking and damaging occurs in a self-similar manner. Thereafter the SST crack growth enters stage IV, called the Tail-End Tearing (TET) fracture. As the crack tips continue to move, they are under the increasing influence of the BSE boundaries $(x= \pm$ $W_{0}$ in Fig. 1a). Finally, the fracture process comes to an end at the instant $f$ of full separation. It should be underlined that the crack growth portion of each test record ( $o-f$ curves in Figs. 2 and 3) cannot be treated as reflecting the real material properties without introducing essential restrictions on the geometries of the specimen and original stress raiser, testing system stiffness, boundary conditions imposed by the grips, loading rate, and fracture process as such.

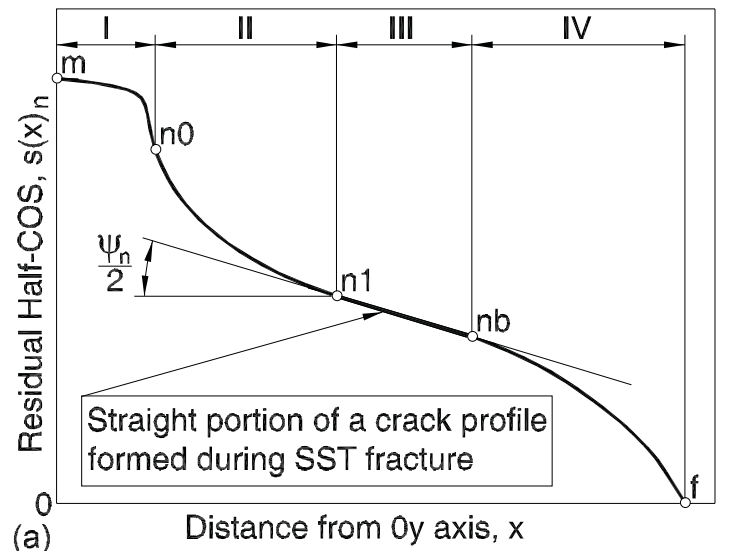

(a)

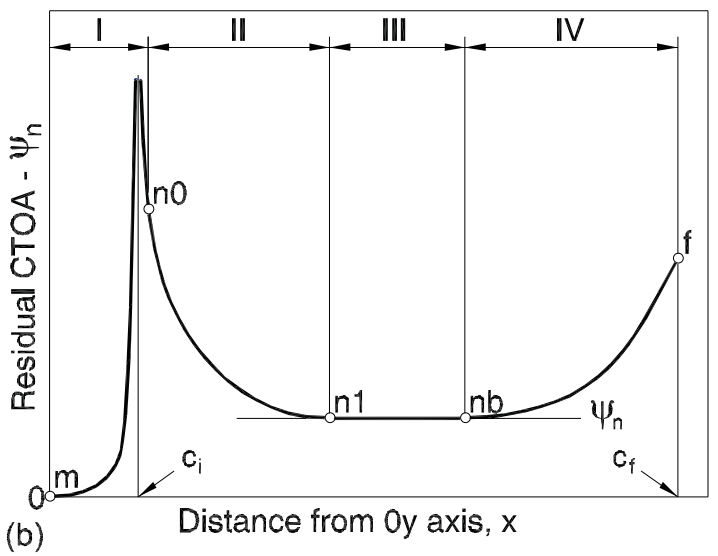

Fig. (3). Schematic presentation of through-life fracture curves expressed in terms of the residual crack opening spacing (a) and residual CTOA (b) determined by moving the upper half of a completely fractured BSE towards its lower one.

In the tests to separation failure, the displacements $v(\mathrm{M})$ and $u(\mathrm{~N})$ of the outer boundaries should increase at a constant 
and extremely small strain rate, i.e., under the quasi-fixed grip condition. This is needed for providing the regime of displacement-controlled tearing from the very instant of NFC nucleation and also for making the SST range of crack extension as wide as possible. The SST portion of the post-test curves ( $n 1-n \mathrm{~b}$ in Figs. 2 and 3 ) denotes the quasi-static fracture process occurring nearly at constant levels of the net-section stresses $\sigma_{\mathrm{N}}$ and $q_{\mathrm{N}}$ (see Fig. 1a), wherein the increments in the Crack Opening Spacing $(\mathrm{COS}), 2 \Delta s(x, c)_{\mathrm{n}}$, are in direct proportion to those in the distance between the virtual crack tips. To establish the SST portion of crack advancements unambiguously, one should consider the through-life fracture curve (Fig. 3a) obtained on a specimen broken down in two identical pieces by a slow stable tear crack. To reduce unavoidable scatter in determining the CTOA angle $\psi_{\mathrm{n}}$ (Fig. 3), two tear cracks of identical geometry must be initiated in a sufficiently large plate from an original stress raiser of a sufficiently small size.

\section{Parameters of a Centre Crack and Fracture Criteria}

The centre crack border provides the basis for much of the following discussion, so it is important to outline the main features of its characterisation. In the current concept of plane stress tearing, the original crack is represented by its planar dimensions irrespective of the spacing $2 s(x, c)_{\mathrm{u}}$ between its upper and lower boundaries in a fully unloaded specimen (compare Figs. 4a and 4b). The crack modelling procedure is specified by the equation $2 a=2 c$, where $2 a$ is the length of a mathematically sharp cut with coinciding surfaces (see straight lines in Figs. 1d and 4a). In the conventional analysis of tearing, the $\operatorname{COS}, 2 s(x, c)$, and the Crack Opening Displacement (COD), $2 v(x, c)$, both being caused by the same applied load, are undistinguishable. Consequently, the crack profile angle $\psi_{\mathrm{c}}$ measured near the crack tip (Fig. 4a) is usually treated as the opening angle, i.e., as an increment in the angular displacement induced by external loading. However, the spacing-based and displacement-based parameters in a real specimen can differ widely, as seen from the scheme in Fig. (4b). It is common knowledge that such differences depend on the specimen geometry and size, type and length of the original stress raiser, boundary restraints, material properties, and loading history. It is pertinent to mention that in the immediate vicinity of the tear crack in a fully unloaded specimen, the material is highly damaged and its behaviour does not respond to the original constitutive relationships.

First, we outline the standard approach to the characterisation of a tear crack profile extending under lowconstraint conditions $[21,22]$. The commonly used fracture criterion states that crack growth occurs when the CTOA- $\psi$ or, equivalently, the $\operatorname{COS}-\delta$ at a fixed distance behind the cracktip attains its critical value $\psi_{\mathrm{c}}$ or $\delta_{\mathrm{c}}$, respectively. Here we consider the determination of the $\psi_{\mathrm{c}}$ angle using optical microscopy to measure the crack contour near its tip (Fig. 4a). Direct measurements of the COS- $\delta$ should be made behind the crack tip and then transformed into the CTOA- $\psi$ values from the following expression:

$\psi_{c}=\frac{1}{N} \sum_{i=1}^{N} \psi_{i}, \psi_{i}=2 \tan ^{-1}\left(\frac{\delta_{c i}}{2 d_{i}}\right)$

Here, $d_{\mathrm{i}}$ is the distance measured behind the crack tip, which ranges from 0.5 to $1.5 \mathrm{~mm}$, and $\delta_{\mathrm{ci}}$ is the related value of the COS. The steady state (average) value of the $\psi$ angle, denoted as $\psi_{\mathrm{c}}$, is established after a minimum amount of crack
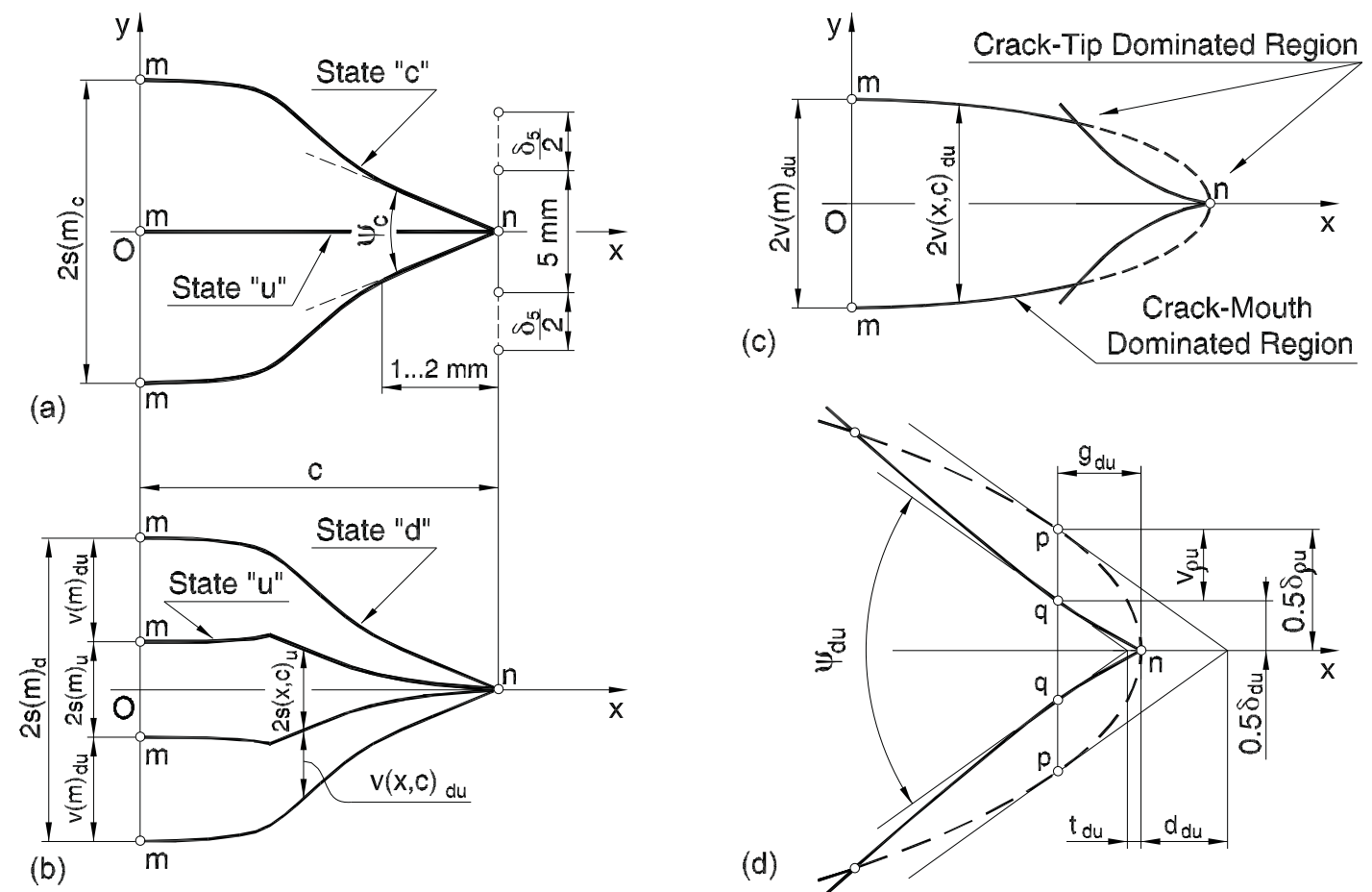

(d)

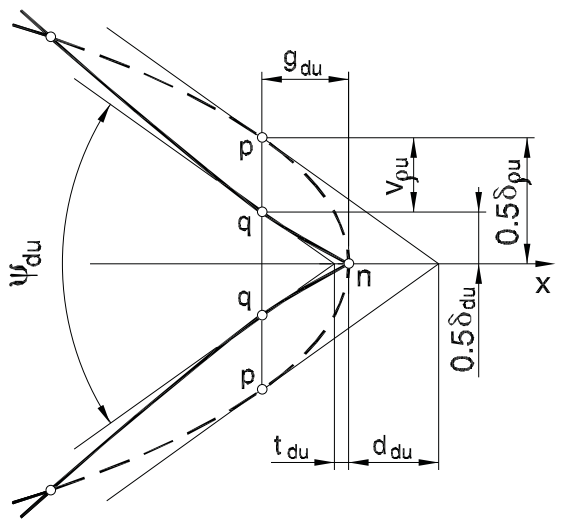

Fig. (4). Definition of the local $\left(\psi_{\mathrm{c}}, \delta_{5}, \psi_{\mathrm{du}}, \delta_{\mathrm{du}}, \delta_{\mathrm{pu}}, t_{\mathrm{du}}, d_{\mathrm{du}}\right)$ and global $\left[c, s(\mathrm{~m})_{\mathrm{c}}, v(\mathrm{~m})_{\mathrm{du}}\right]$ parameters of the tear crack profile used in the current (a) and in the UM (b), (c), and (d) procedures for characterising the fracture resistance during the tear crack extension (all variables are not in scale). 
extension $\Delta c_{\min }$. The latter corresponds to the instant where the angle $\psi$ attains a nearly constant level. If the size requirements of the standards $[21,22]$ are met, then $\psi_{\mathrm{c}}$ is deemed to characterise the material in the thickness of a specimen tested and to be independent of the in-plane geometry and loading scheme. The practicality and usefulness of this formulation of the fracture criterion for predictions of tearing in airframe materials was demonstrated in many experimental and numerical studies, e.g., those performed by Newman [35-37].

Alternatively, the $\delta_{5}-\mathrm{R}$ curve concept developed by Schwalbe [38] can be used for an approximate determination of the CTOA. This concept has a global nature with respect to a growing crack and requires much less effort than the previous one to experimentally determine the $\psi_{\mathrm{c}}$ angle. It provides a relatively simple method because the measurement points should not migrate with the moving crack tip. The CTOD- $\delta_{5}$ is given by the relative displacement $\delta_{5}$ between a pair of points on either side of the initial fatigue crack, as shown in Fig. (4a). In the initial range of crack extension, the $\delta_{5}$-R curve can be approximated by a power-law regression fit of the experimental measurements. In this way the CTOD- $\delta_{5}$ values related to the values $\Delta c$ of advancement of one crack tip are used to calculate the CTOA according to the relationships

$\delta_{5}=D \Delta c^{p}, \psi=\tan ^{-1}\left(\frac{d \delta_{5}}{d \Delta c}\right) \approx \frac{d \delta_{5}}{d \Delta c}=D p \Delta c^{p-1}$

Our concept of plane stress tearing is based on presenting the profile of a central crack in terms of the $\operatorname{COD}-v(x, c)_{\mathrm{du}}$, i.e., distances between the mating points on the crack border for a loaded (state $d$ ) and fully unloaded (state $u$ ) specimen (Figs. 2 and $\mathbf{4 b}$ ). For a BSE of a ductile material, it may be described by simple model equations (well-defined curves). The first curve encompasses the socalled crack-mouth dominated region and the other the crack-tip dominated region, as indicated in Fig. (4c). Their intersection denotes an imaginary transition from the convex to concave portions of the crack profile. In an actual specimen, these portions are connected by a smooth curve of a transient behaviour. The length of the crack-tip dominated region in brittle materials is negligibly small, compared with the length of the crack-mouth dominated region for the same crack.

First, we consider the UM approach as applied to modelling a tear crack in the BSE of a homogeneous linear elastic material. For this simplest case, the following hypothesis was taken as a starting point. The physical essence of the fracture micro-mechanisms within the ADZ is independent of the sign and value of the stress biaxiality ratio $k$. This hypothesis suggests that the model descriptions of fracture in tension-dominant and compressiondominant crack geometries are conceptually identical. A simple and still physically relevant approach is to treat the SST as a two-directional extension of an ideal crack in the form of an open elliptic hole (Fig. 1e). Both tips of this hole in a stress-free BSE made from a given material of a given thickness have identical curvature radii of the surface $\rho=b^{2} / c$, where $b$ and $c$ are the minor and major semi-axes of this imaginary hole.
The radius $\rho$ is not a directly measurable physical quantity, but a mathematical abstraction quantitatively characterised by the following equation:

$\rho_{d u}=\frac{v(m)_{d u}^{2}}{c}=\rho \cdot \frac{\left\{1+C_{n}\left(\sigma_{d u} / E\right)\right\}^{2}}{\left\{1+C_{m}\left(\sigma_{d u} / E\right)\right\}}$

where $C_{\mathrm{n}}$ and $C_{\mathrm{m}}$ are the stress concentration factors at the points $x= \pm c, y=0$ and $x=0, \mathrm{y}= \pm b$, respectively. They take the form

$C_{n}=F_{v} \cdot\left[1+2 \sqrt{\frac{c}{\rho}}-k\right]$ and $C_{m}=F_{u} \cdot\left[k+2 k \sqrt{\frac{\rho}{c}}-1\right]$.

In the case of a BSE with free-to-move boundaries, the dimensionless elastic compliances of the crack border along the transverse, $F_{v}=v(x=0, k) \cdot E /\{b(1-k)+2 c\} \cdot \sigma$, and longitudinal, $\quad F_{u}=u(x=c, k) \cdot E /\{k(c+2 b)-c\} \cdot \sigma \quad$ directions depend only upon the dimensionless parameters $\rho / c$, $c / W_{0}$, and $H_{0} / W_{0}$. Taking into account that the $F_{\mathrm{v}} / F_{\mathrm{u}}$ ratio does not vary with the stress-biaxiality ratio $k$, one can define the $\rho$ radius from equation (3) using the $v(\mathrm{~m})_{\mathrm{du}}$ values measured on a BSE-type specimen under uniaxial tension.

Due to the interplay between the moving crack tips and the outer specimen boundaries, the $\rho$ value depends upon the aforementioned dimensionless parameters. The initial radius $\rho$ $=\rho_{\mathrm{f}}$ is treated as a characteristic of an SST crack of any length in a stress-free BSE of a given material, plate thickness, and loading scheme. The critical radius of an imaginary elliptic hole corresponds to the geometry of an actual crack extending in the SST regime, when $\rho_{\mathrm{du}}=\rho_{\mathrm{d}}$. Here, we assume that the radius $\rho_{\mathrm{d}}$ is an inherent length scale of an ideally brittle material characterising the resistance to SST crack growth in a BSE at a given level of in-plane constraint measured by the $k$ ratio. Thus, the UM criterion of SST fracture at a fixed value of the stress biaxiality ratio $k$ requires that the conditions $\rho=\rho_{\mathrm{f}}$ and $\rho_{\mathrm{du}}=\rho_{\mathrm{d}}$ be met simultaneously.

In order to assess the ductile tearing resistance under plane stress conditions, a set of additional assumptions was incorporated in the UM fracture analysis. They can be formulated as follows: (i) There are no intrinsic differences between the micromechanical behaviour of a material during the accumulation of diffused damage, localisation of plastic deformation, nucleation of an NFC, and its stable propagation; (ii) Once the peak levels of strain hardening and diffused damage are attained, the incubation and accumulation of localised damage near the stress-free crack surfaces manifest themselves mainly as an increase in the crack-border spacing; and (iii) The behaviour of the SST crack under general yielding reflects a specific interplay between the inner and outer boundaries of a cracked plate.

The latest (updated) version of the multi-parameter characterisation of near crack-tip profiles is sketched in Fig. (4d). All geometric variables in question emerge from the joint consideration of two model equations:

$$
\begin{aligned}
& v(x, c)_{d u}=v(m)_{d u} \sqrt{1-x^{2} / c^{2}} \\
& v(x, c)_{d u}=G \cdot(c-x)^{F}
\end{aligned}
$$


that describe geometries of the crack-mouth and crack-tip dominated regions, respectively. Taking the derivatives of these expressions with respect to the distance $x$ from the $0 y$ axis (Fig. 4c) and upon equating them, one can define the characteristic points on the near crack-tip profile. These are two pairs of points $p$ and $q$ behind the crack tip where the CTOA- $\psi$ and COD- $\delta$ both attain their critical values $\psi_{\mathrm{du}}$ and $\delta_{\mathrm{du}}$, respectively. When taken together with the global crack profile parameters (Fig. 2), the crack-tip parameters shown in Fig. (4d) can be used in assessing the ductile tearing resistance. Despite the fact that the UM uses a large number of geometric parameters, all of them are directly related to each other and also to the data of macroscopic measurements.

To be in line with the standards [21, 22], the UM fracture criterion is formulated solely in terms of the CTOA- $\psi$ parameter. We hope that the latter has a great deal of physical significance because the geometry of the crack-tip dominated region results from direct measure- ments. It is assumed that the critical state of an extending tear crack is reached at the instant when its tips enter the SST range of crack advancement (point $s 1$ in Fig. 2). This fracture event corresponds to the instant where the minimum value $\psi_{\mathrm{n}}$ of the residual angle $\psi_{\text {res }}$ (point $n 1$ in Fig. 3b) and the maximum value of the active component of the CTOA- $\psi_{\mathrm{du}}$, denoted $\psi_{\mathrm{d}}$, are attained simultaneously. Finally, the UM criterion of ductile tearing is given by two conditions:

$\psi_{\text {res }}=\psi_{n}$ and $\psi_{d u}=\psi_{d}$

\section{Scope of Study and Specimens}

This paper deals with the characterisation of plane stress tearing under uncontained yielding in flat specimens (Fig. 5) made from thin sheets of an aircraft-skin aluminium alloy. The principal obstacle to the development of an easy-to-use TL is placed by the need to correlate too many variables governing the plane-stress crack growth in ductile materials. These are the parameters of elasticity, including those of out-of-plane (a)

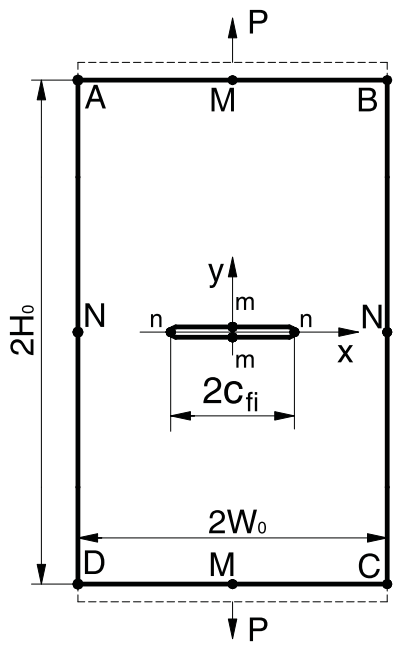

(b)

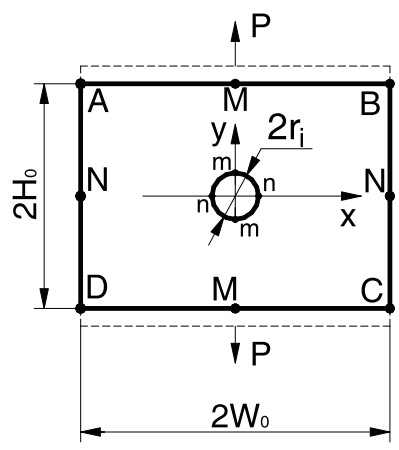

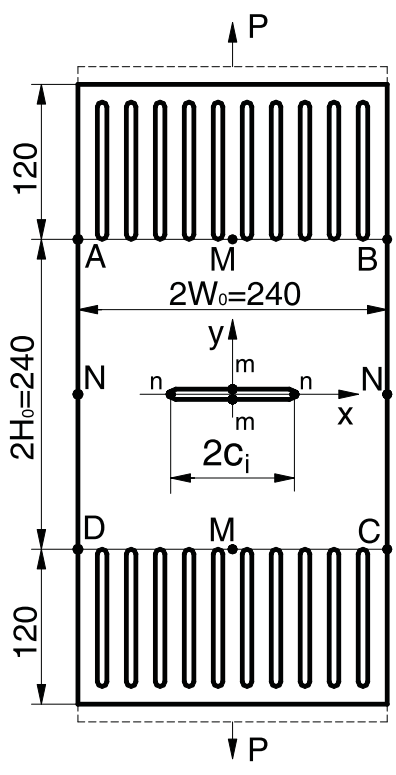

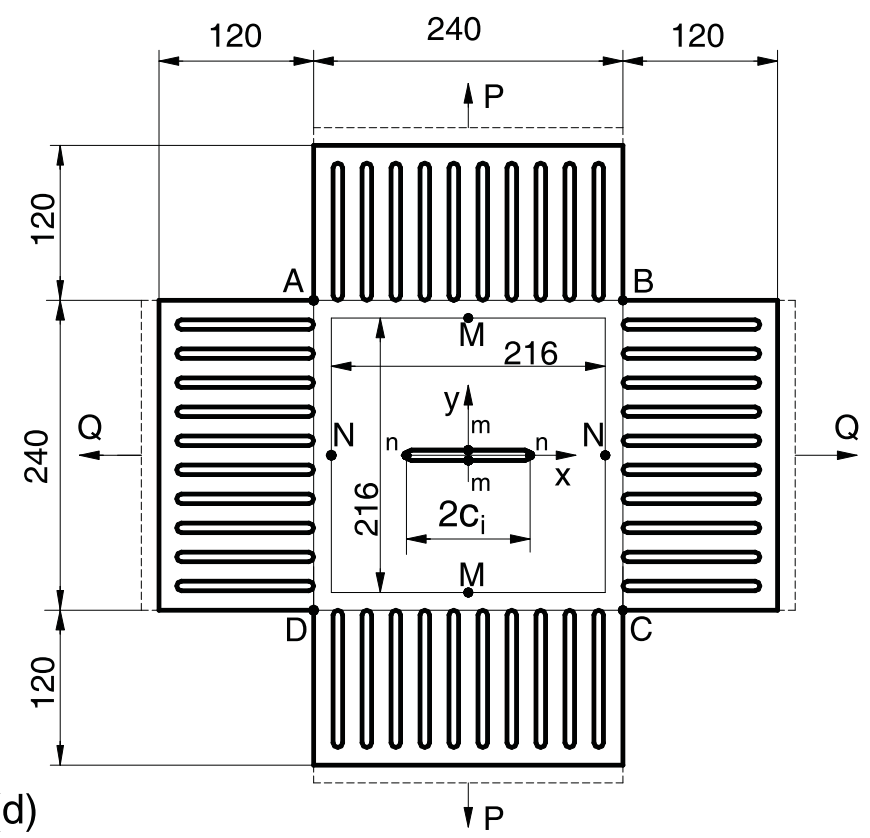

Fig. (5). Problem domains $A B C D$ attached to different loading fixtures referred to as (a) $-\mathrm{M}(\mathrm{T}),(\mathbf{b})-\mathrm{MR}(\mathrm{T}),(\mathbf{c})-\mathrm{MM}(\mathrm{T})$, and (d) MM(T-TC) specimens (the dimensions are indicated in millimetres). 
deformation (buckling); plasticity, including those of residual stress effects and anisotropy; geometric and structural imperfections; diffused and localised necking; damage and cracking. So it seems highly improbable that the stable crack growth could be predicted using only the near crack-tip parameters in isolation from the global deformation pattern and localised necking.

The problem under consideration is addressed in two parts. We start with comparing the profiles of tear cracks in loaded and in fully-unloaded specimens of different geometries under different boundary conditions. The intent is to reveal the basic distinctions between the current and the UM approaches to analysing changes in the overall geometry of a stationary and an extending tear crack in the BSE. In the second part of this work, different characterisations of plane stress tearing are contrasted with each other. The objective is to determine whether they can or cannot assess the SST state of a crack growing under different in-plane constraint states. To obtain conclusive evidences, we tested specimens of widely different shapes, sizes, and loading scheme.

The need for a better understanding of the reason for the gap between the results of model descriptions and measurements dictates the breadth of experimental efforts. Therefore, the fracture tests in question are meant to give the "big picture" of structural behaviour of a middlecracked plate under different constraint states (Fig. 5). The uniaxial and biaxial crack extension tests were performed on flat specimens with Problem Domains (PD) of various shapes and sizes given in Table 1. Each rectangular PD was attached to a specific loading fixture.

Table 1. Principal Dimensions of Specimens

\begin{tabular}{|c|c|c|c|c|}
\hline Specimen code $^{\mathbf{a}}$ & $\begin{array}{c}\mathbf{2} \boldsymbol{W}_{\mathbf{0}} \\
\mathbf{m m}\end{array}$ & $\begin{array}{c}\mathbf{2} \boldsymbol{H}_{\mathbf{0}} \\
\mathbf{m m}\end{array}$ & $\begin{array}{c}\mathbf{2} \boldsymbol{H}_{\mathbf{p}}\left(\mathbf{2} \boldsymbol{W}_{\mathbf{q}}\right) \\
\mathbf{m m}\end{array}$ & $\begin{array}{c}\mathbf{2} \boldsymbol{c}_{\mathbf{i}} \\
\mathbf{m m}\end{array}$ \\
\hline \hline $\mathrm{MM}(\mathrm{T}-\mathrm{TC})-1.0-2.0$ & 240 & 240 & $480(480)$ & 30 \\
\hline $\mathrm{MM}(\mathrm{T})-1.0-2.0$ & 240 & 240 & 480 & Variable \\
\hline $\mathrm{M}(\mathrm{T})-3.0-10.0$ & 1200 & 3600 & 3600 & 60 \\
\hline $\mathrm{M}(\mathrm{T})-1.0-10.0$ & 1200 & 1200 & 1200 & Variable \\
\hline $\mathrm{MR}(\mathrm{T})-1.0-1.0$ & 120 & 120 & 120 & 2.0 \\
\hline $\mathrm{MR}(\mathrm{T})-0.1-1.0$ & 120 & 12 & 12 & 2.0 \\
\hline
\end{tabular}

${ }^{a}$ The numerical values in the specimen code denote the shape ratio $\left(H_{0} / W_{0}\right)$ and scale ratio $\left(W_{0} / W_{0}{ }^{\mathrm{BSE}}\right)$, respectively. In this work we take $2 W_{0}^{\mathrm{BSE}}=120 \mathrm{~mm}$.

It should be emphasised that providing a sufficiently high degree of uniformity of nominal stress fields in tension- and/or compression-dominant PDs is essential for collecting acceptable test data. In the case of tensile loading, this can be achieved through increasing the aspect ratio $H_{0} / W_{0}$ for the $\mathrm{M}(\mathrm{T})$ specimen (Fig. 5a). However when the PD geometry and size are fixed, some special modifications in the loading fixture design must be used $[39,40]$. One of them is applying the external load through thin strips placed between the PD and the grips. These strips in specimens of thin-sheet materials are usually cut with saw, as shown in Figs. (5c) and (5d).
The larger is the number of slots in each side of the fixture and the thinner and longer they are, the larger the size of a uniformly stressed region. This region was defined for a crackfree PD with the use of the photoelastic and, in parallel, elastic finite element analyses [39]. The accepted number of the slots of length equal to $0.85 W_{0}$ in each side of the $\mathrm{MM}(\mathrm{T}-\mathrm{TC})$ specimen was 15 . In this case, the width and height of the uniformly stressed region reach 0.9 of the PD dimensions $2 W_{0}$ $=2 H_{0}=240 \mathrm{~mm}$. The deviation of the peak values in the nominal stress distribution from the average values of the elastic stresses $\sigma(x)=P / 2 W_{0} B_{0}$ and $q(y)=Q / 2 H_{0} B_{0}$ does not exceed $2 \%$ within the uniformly stressed region. There are good reasons to suggest that in our specimens the uniformly stressed region is larger than that in typical specimens used in biaxial fracture tests. References [18, 41-44] show an example.

Consider unavoidable distinctions between the boundary conditions for the PDs of the cruciform specimen (Fig. 5d) and the BSE (Fig. 1a). The lack of restraints imposed by an actual loading fixture on the deformation of an actual PD is seldom if ever in occurrence. For typical combinations of the specimen and grips, exact stress and displacement conditions along the boundaries between the middle-cracked PD and the loading fixtures are usually nonuniform and unknown. Generally, both stress conditions and displacement conditions are present. Under mixed boundary conditions the applied stress is a function not only of position, but also of the crack aspect ratio $c / W_{0}$ and shape ratio $H_{0} / W_{0}$. Mixed boundary conditions are part way towards the general compliant boundary conditions. Such is indeed the case for the elastic-plastic behaviour of the specimens shown in Figs (5c) and (5d).

When the restraints imposed on the outer boundaries are close to the case of displacement-controlled loading, the fracture response strongly depends on the crack length both in the quantitative and qualitative senses [45]. Earlier it was shown [15] that the asymptotic values of the energy dissipation rate $R$ for the MM(T) and MM(T-TC) specimens (Fig. 5) made of low-carbon steel are substantially different. On the other hand, the imposition of the "fixed-grip" and "dead-load" constraints on the outer BSE boundaries may have the effect of eliminating the presence of the remote load $q$ in the energy rate calculations [18].

In our tests we used three types of loading fixtures. The first one provides rigid clamping along the horizontal boundaries of a PD (Figs. 5a and 5b). If the shape requirements $H_{0} \geq 2 W_{0}$ and $c \leq W_{0} / 3$ are fulfilled and buckling is prevented, the geometry in Fig. (5a) is usually referred to as the standard $\mathrm{M}(\mathrm{T})$ specimen. The second loading fixture ensures a nearly uniform distribution of the nominal tensile stress $\sigma(x)$ on the horizontal boundaries of a PD with no initial crack. This geometry (Fig. 5c) is referred to as the $\mathrm{MM}(\mathrm{T})$ specimen. It is rigidly clamped along the lines $y= \pm H_{\mathrm{p}}$, where $H_{\mathrm{p}}=\left(H_{0}+120\right) \mathrm{mm}$. Finally, the third fixture in combination with a square $\mathrm{PD}$ represents a cruciform specimen (Fig. 5d) designated as $\mathrm{MM}(\mathrm{T}-\mathrm{TC})$. When $2 c_{0}=0$, nearly uniform stresses $\sigma(x)$ and $q(y)$ prevail on the horizontal and vertical boundaries of the given PD, respectively. The specimen is rigidly clamped along the lines $x= \pm W_{\mathrm{q}}$ and $y= \pm H_{\mathrm{p}}$. The MM(T-TC) geometry is treated as the physical counterpart of the BSE geometry.

A centred starting slot of length $2 c_{\mathrm{i}}$ was made in each specimen of the $\mathrm{M}(\mathrm{T}), \mathrm{MM}(\mathrm{T})$ and $\mathrm{MM}(\mathrm{T}-\mathrm{TC})$ geometry by 
manual cutting. The depth of cut was kept to the practical minimum for a jeweller's saw. All slots had nominally straight and parallel flanks with the root radii $r_{\mathrm{i}}$ less than $0.06 \mathrm{~mm}$. A single circular hole of diameter $2 r_{\mathrm{i}}=2 \mathrm{~mm}$ was introduced in each MR(T) specimen (Fig. 5b). This hole is treated as a small geometric imperfection ensuring that the fracture process occurs only by the mechanism of shear localisation $[32,33]$.

The specimens $\mathrm{M}(\mathrm{T})-3.0-10.0, \mathrm{M}(\mathrm{T})-1.0-10.0, \mathrm{MR}(\mathrm{T})-$ 1.0-1.0, and MR(T)-0.1-1.0 shown in Fig. (5) and Table 1 are considered to be more constrained than the others. The low-constrained specimens include the $\mathrm{MM}(\mathrm{T})-1.0-2.0$ specimens tested along (LT direction) and across (TL direction) the rolling direction. Several MR(T)-1.0-1.0 specimens were also tested along and across the rolling direction.

\section{MATERIAL AND TESTING}

The test material is aluminium alloy D16AT in asreceived condition, having the form of $1.4-1.5 \mathrm{~mm}$ thick sheets. Its chemical composition and mechanical properties are close to those of AL 2024-T3. In particular, the properties of AL-alloy D16AT under ambient conditions were determined in tensile tests using the standard platetype specimens are as follows: the elastic modulus $E=$ $67.7 \mathrm{GPa}$, Poisson's ratio $v=0.32$, the $0.2 \%$ offset yield strength $\sigma_{\mathrm{Y}}=338 \mathrm{MPa}$, and the ultimate tensile strength $\sigma_{\text {UTS }}=465 \mathrm{MPa}$. Three identical specimens were fabricated and tested in accordance with the ASTM Standard Method for Tension Testing of Metallic Materials (E8M-85). These specimens were subjected to displacement-controlled loading (with the rate $0.017 \mathrm{~mm} / \mathrm{s}$ ) in direction parallel to the rolling direction of the sheets.

In collecting test data, a purely mechanistic approach based on the minimum of assumptions was adopted. Uniaxial and biaxial tests were conducted in accordance with the main requirements of the ASTM Standard Practice for R-Curve Determination (E561-92a). All specimens (see Table 1), excluding those of the MR(T) type, were tested with guide plates lightly clamped against the out-of-plane displacement. Buckling of thin plates is a competitive failure mechanism resulting from the elastic compressive stress acting parallel to the crack growth line. We deem that the various guide plate systems used in our tests do allow decoupling the fracture process from the buckling. However, the stiffness of these plates was different and unknown. It must be noted here that in wide panel tests conducted by Dawicke et al. [46] the load versus crack extension diagram strongly depends on the stiffness of the guide plate system. Our measurements of the out-of-plane displacements in tests of the MR(T)-1.0-1.0 specimens demonstrate that the buckling is negligibly small when the condition $c \leq 0.4 W_{0}$ is fulfilled.

During displacement controlled tests, the $\mathrm{MM}(\mathrm{T})$ and $\mathrm{MM}(\mathrm{T}-\mathrm{TC})$ specimens of the first set were loaded incrementally, allowing some time between steps for the crack to stabilise before measuring the load, crack length, and crack profile. The MR(T) specimens were tested under monotonically increasing displacement $v(\mathrm{M})$ (with the rate $0.001 \mathrm{~mm} / \mathrm{s}$, without stopping and unloading). Displacements as a function of the proportionally applied loads $P$ and $Q$ were measured concurrently at the extreme points on the inner and outer PD boundaries that are shown in (Fig. 5). To develop $R$-curves with confidence, we usually assigned more than ten steps (data points) for each test condition. Once the crack stabilised within seconds after the loading was stopped, a close-up photograph of the crack-tip profile was taken. In most cases, four diagrams were recorded simultaneously, namely, $P v s .2 v(\mathrm{~m}), P v s$. transverse displacement $2 v(\mathrm{M}), P$ vs. load point displacement $2 v(\mathrm{P})$, and $P$ vs. displacement $2 u(\mathrm{~N})$.

All the measurements mentioned above were repeated for the rest of these specimens containing an arrested tear crack after they were completely unloaded. The specimens were strained very slowly to approximate the quasi-fixed grip conditions. Thus, the fracture process could be readily stopped by the termination of loading with or without subsequent unloading. The above measurements were repeated once again at the instant $t$ when the loading was stopped and then after the complete unloading at the instant $u$. Typical diagrams are shown in Figs. (6-8). In processing test data, the state $t$ is treated as the state $d$ on the damaging curve $(i-d-f)$ shown in Fig. (2).

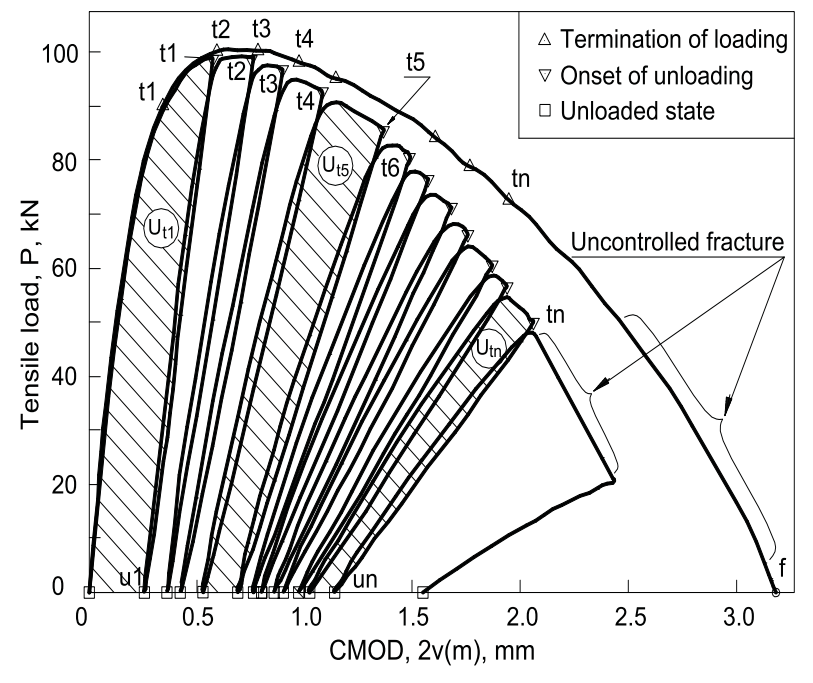

(a)

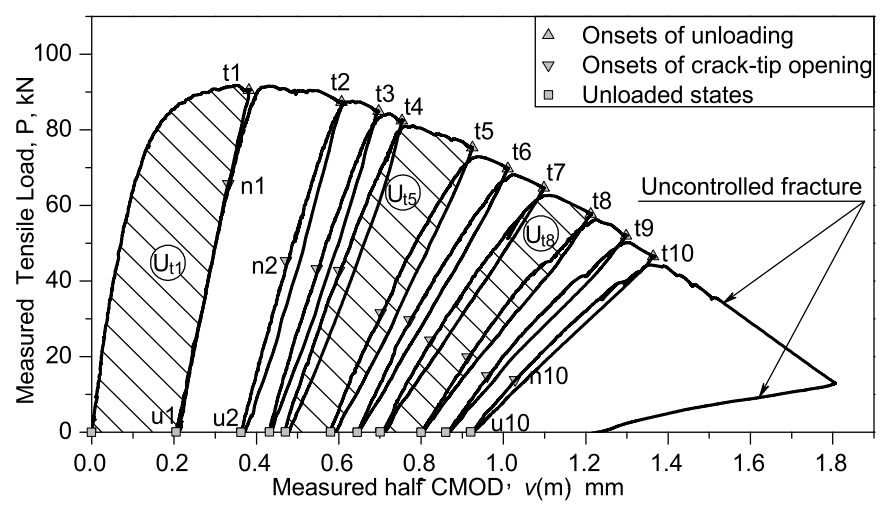

(b)

Fig. (6). $P-2 v(\mathrm{~m})$ test records for two MM(T-TC)-1.0-2.0 specimens (a) and an $\mathrm{MM}(\mathrm{T})-1.0-2.0$ specimen (b), all tested in the LT direction. The cruciform specimens were tested in proportional biaxial tension at a constant value of load biaxiality ratio $k=0.8$. 

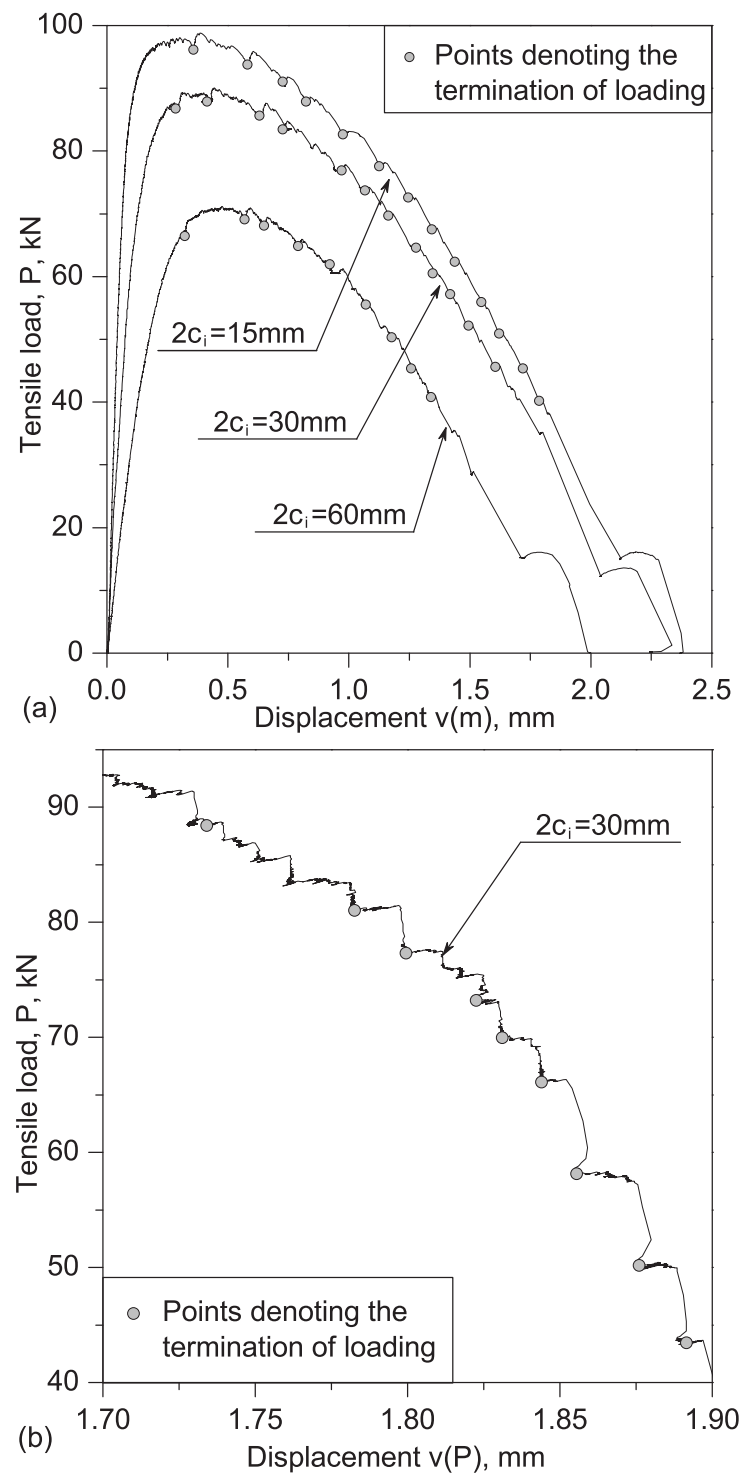

Fig. (7). Test records of tensile load $v s$. displacements $v(\mathrm{~m})$ measured near the points $m$ for $\mathrm{MM}(\mathrm{T})-1.0-2.0$ (TL direction) specimens (a) and displacements $v(\mathrm{P})$ measured near the points $P$ of load application for $\mathrm{MM}(\mathrm{T}-\mathrm{TC})-1.0-2.0$ (LT direction) specimens (b).

\section{EXPERIMENTAL RESULTS}

First, we consider a set of data obtained from direct measurements of the crack profiles in PDs of different shapes, sizes, and loading scheme (Figs. 9-17). Taken together, they firmly support the UM approach to characterizing the tear crack profile in the BSE. One can see that the crack-mouth compliances calculated from the Eftis-Liebowitz equation [47] are lower than the measured values (Figs. 9 and 10). This equation was included in the ASTM standard (E561-92a) in spite of the fact that in the literature, empirically determined crack-mouth compliances are always higher than those from the above equation. Schijve points out in [48] that the discrepancies vary from a few percent to 11 percent. They should not be due to the approximate character of the equation, as exemplified by the finite element analysis presented in [49]. The situation is so much unsatisfactory because the above discrepancies could not be explained by the experimental inaccuracies of measurements either. But much more important is the fact that the equation in question has no relevance to the tear crack profiles in loaded specimens (states $t$ in Figs. 10 and 12).

The above results are helpful for understanding the UM procedure for displacement-based assessment of plane stress tearing resistance. Initially, one has to determine a relationship between the tear crack length $2 c$ and the tensile load $P$ (Fig, 18). In processing, test data are averaged and then approximated by cubic polynomials. Next step consists in polynomial fitting of the test data on the specimen compliance (Fig. 19). Two relationships in question allow correlating the crack length $2 c$ with the CMOD $-2 v(\mathrm{~m})_{\mathrm{du}}$. Thus, one can determine the values $2 v(\mathrm{~m})_{\mathrm{du}}$ for any crack length within the range $c_{\mathrm{i}} \leq c<W_{\mathrm{f}}$, as it is shown in Fig. (20). And finally, these through-life fracture curves are used together with equations (3), (4), and (5) in accordance with the aforementioned explanations. The effect of load biaxiality on the geometric parameters characterising the near crack-tip profile is displayed in Figs. (21), (22), and (23).

To support the UM presentation of the through-life fracture behaviour (Fig. 2), we consider test records obtained on specimens of the MM(T) and MM(T-TC) types (Figs. 6, 7, and 8). Tear cracks were growing under well-developed or general yielding in all specimens (see as an example Fig. 24), excluding those of the width $2 W_{0}=1200 \mathrm{~mm}$. The tear crack

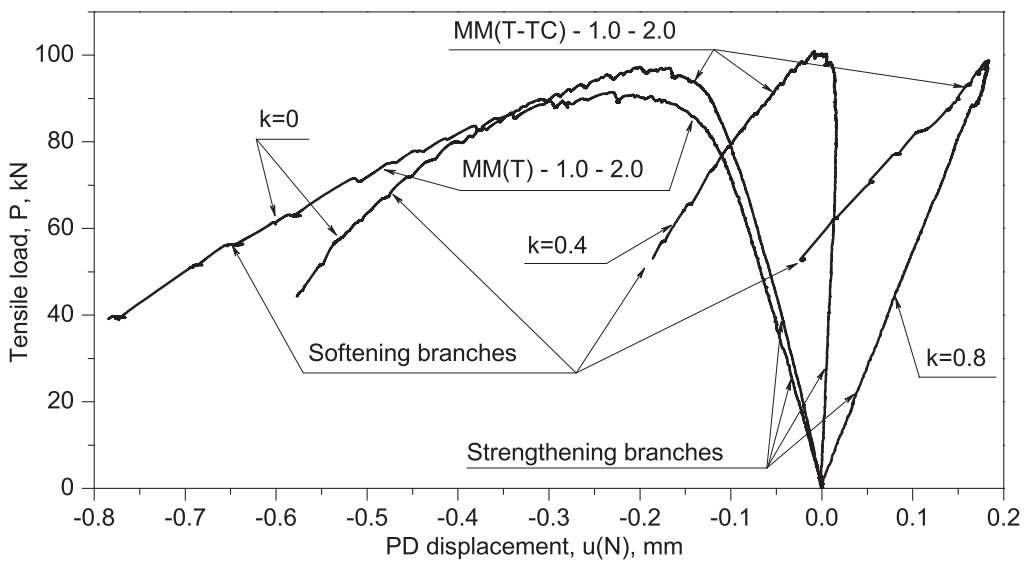

Fig. (8). $P-u(\mathrm{~N})$ test records for $\mathrm{MM}(\mathrm{T})-1.0-2.0$ and $\mathrm{MM}(\mathrm{T}-\mathrm{TC})-1.0-2.0$ specimens (LT direction) containing an identical stress raiser of length $2 c_{\mathrm{fi}}=30 \mathrm{~mm}$ and radius of its tips $r_{\mathrm{fi}} \approx 0.06 \mathrm{~mm}$. The specimens were loaded in uniaxial and proportional biaxial tension at different $k$ ratios using the antibuckling guide plates. 


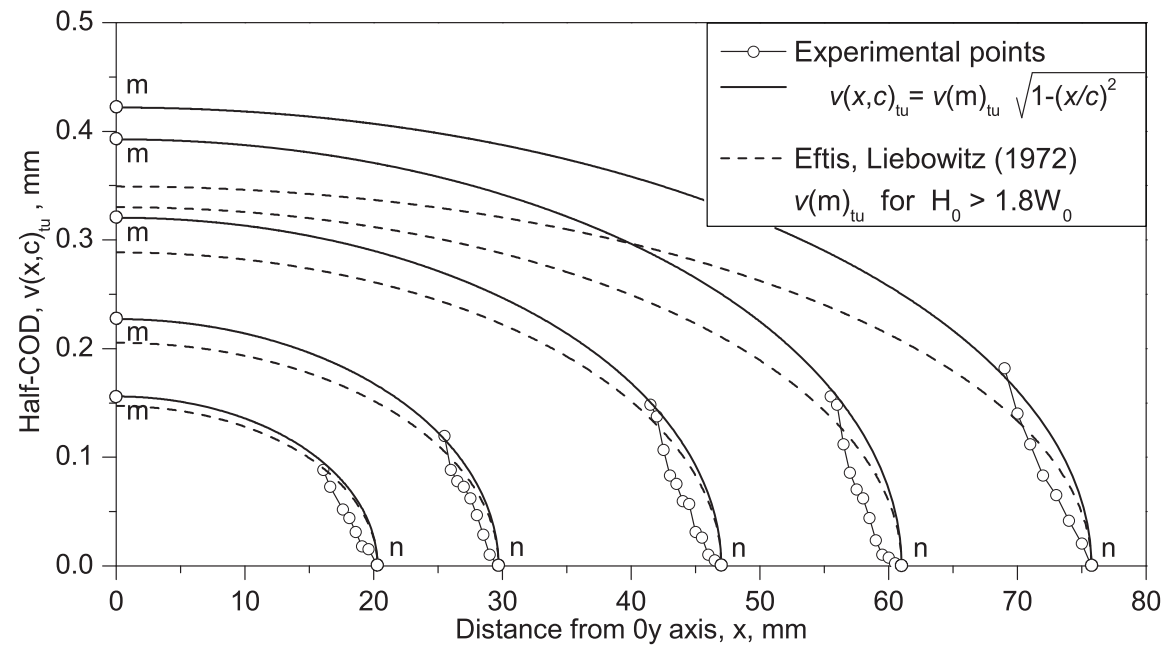

Fig. (9). Comparison of the elliptic crack profiles expressed in terms of the opening displacements, which are constructed using the directly measured (solid lines) and calculated (dashed lines) values of $v(\mathrm{~m})_{\mathrm{tu}}$ for an MM(T)-1.0-2.0 specimen (LT direction). The global profiles (solid lines) are matched with the corresponding displacements measured near the crack tip.

extension occurs by an intermittent attainment of the local instabilities displayed graphically in Figs. (7) and (8). These observations are in accord with the arguments of Turner and Kolednik [50], who believe that the crack growth at both micro- and macro-levels may be seen as a two-stage process of damage accumulation in a process zone followed by the actual separation being a micro-

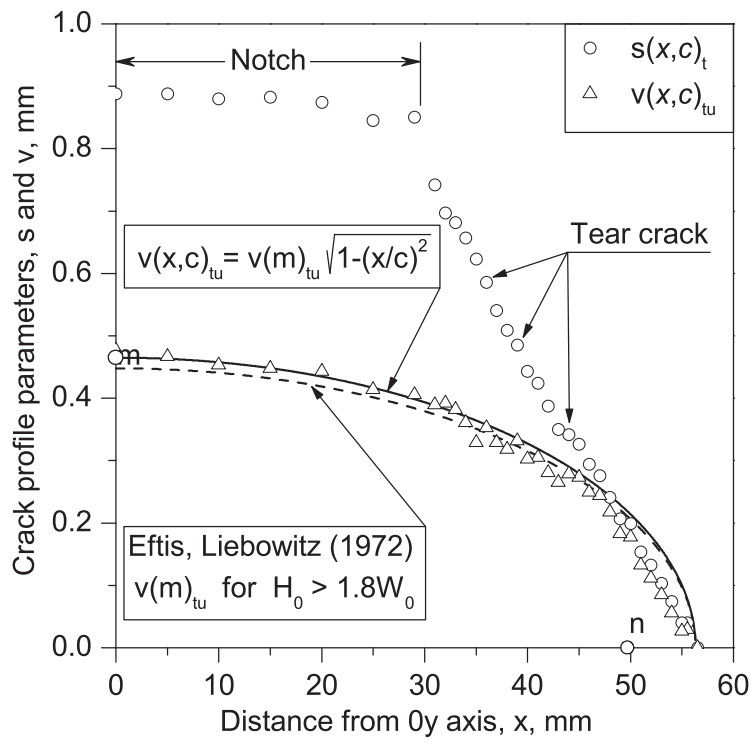

Fig. (10). Experimental data on the opening spacing $s(x, c)_{\mathrm{t}}$ for a stress raiser in a large-scale $\mathrm{M}(\mathrm{T})-3.0-10.0$ specimen of width $2 W_{0}=1200 \mathrm{~mm}$ at the termination of loading (state $t$ ) and an elliptic curve (solid line) approximating the distribution of the inplane displacements $v(x, c)_{\mathrm{tu}}=s(x, c)_{\mathrm{t}}-s(x, c)_{\mathrm{u}}$, where $s(x, c)_{\mathrm{u}}$ is the opening spacing after the complete unloading. The elliptic crack profile (dashed line) was calculated assuming the linear elastic behaviour of the actual crack modelled by a mathematical cut of the length $2 a=2 c$.

instability at the crack tip. It is pertinent to cite here the following conclusion of Ming $\mathrm{Li}$ and Richmond [51]: "In the hardening regime, plastic or inelastic deformation at modest strain is intrinsically unstable and nonuniform and develops in small temporally confined discontinuous jumps." Therefore, the SST is treated in the UM as a step-wise enlargement of the crack cavity bounded by $i-s-f$ and $i-d-f$ curves shown in Fig. (2).

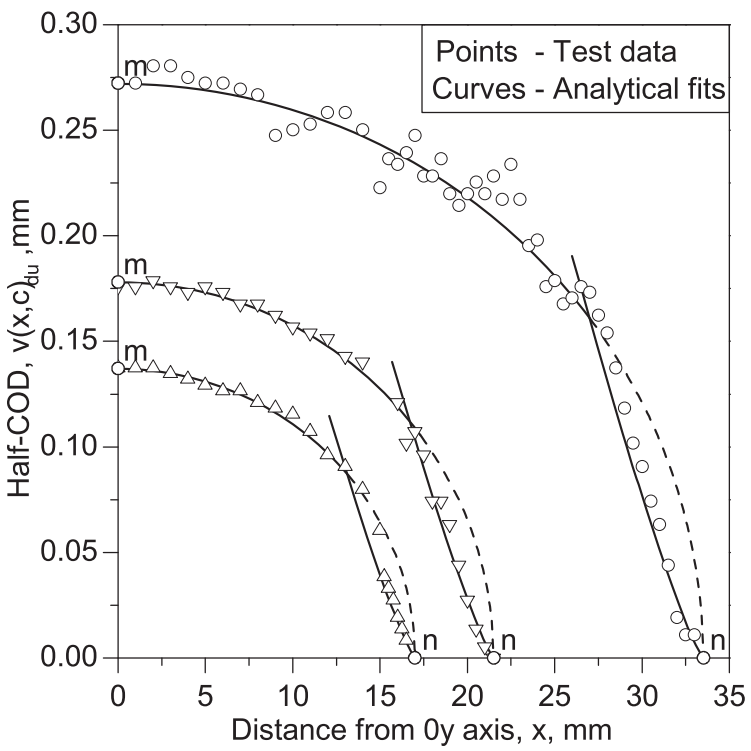

Fig. (11). Experimental data on the opening displacement $v(x, c)_{\mathrm{du}}$ for one-quarter profile of the same tear crack in an MM(T-TC)-1.0-2.0 (LT direction) specimen after three cycles of unloading-reloading at $k$ $=0.4$.

\section{DISCUSSION}

The need to include the crack-tip length scales in the fracture mechanics analyses has long been felt and has been increasingly urgent. In studies of plane stress tearing, the commonly-used length scale is presented by the radius $r_{0}$ of a zone with a highly deformed or damaged material defined by the simplifying relationship

$r_{0}=\frac{1}{\pi} \cdot\left(K_{c} / \sigma_{0}\right)^{2}$ 
where $K_{\mathrm{c}}$ is the fracture toughness of the material and $\sigma_{0}$ is its characteristic strength under uniform tensile loading. The value $r_{0}$ is considered to be the intrinsic characteristic of a material because both quantities $K_{\mathrm{c}}$ and $\sigma_{0}$ are usually taken as physical constants, i.e., material properties. In fact, these assumptions have no definite physical meaning and reflect widespread oversimplifications often used to perform engineering calculations.

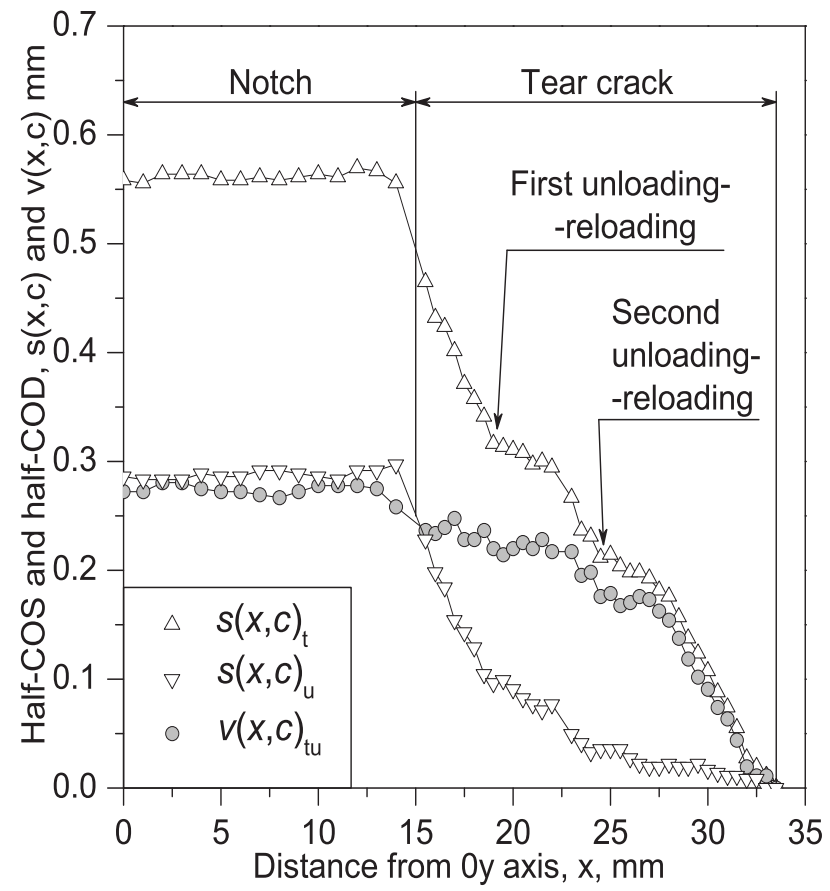

Fig. (12). Experimental data on the opening spacing $s(\mathrm{x}, c)$ for one-quarter of the original stress raiser in an MM(T-TC)-1.0-2.0 (LT direction) specimen at the termination of loading (state $t$ ) and full unloading (state $u$ ) and the opening displacement $v(\mathrm{x}, c)_{\mathrm{tu}}$ defined as the difference $s(\mathrm{x}, c)_{\mathrm{t}}-s(\mathrm{x}, c)_{\mathrm{u}}$. The stress raiser comprises a centre notch and two tear cracks grown-up at $k=0.4$ with three cycles of unloading-reloading.

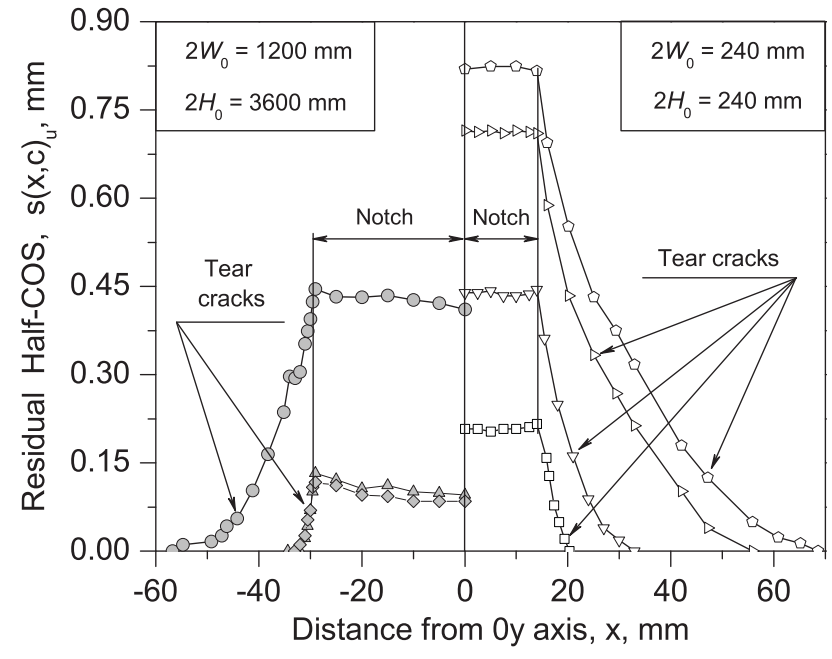

Fig. (13). Comparison of one-quarters of stress raisers in fully unloaded $\mathrm{M}(\mathrm{T})-3.0-10.0$ (left side) and $\mathrm{MM}(\mathrm{T})-1.0-2.0$ (right side) specimens showing very different values of irreversible displacements for raisers of the same length in low-constraint PDs with widely different sizes.
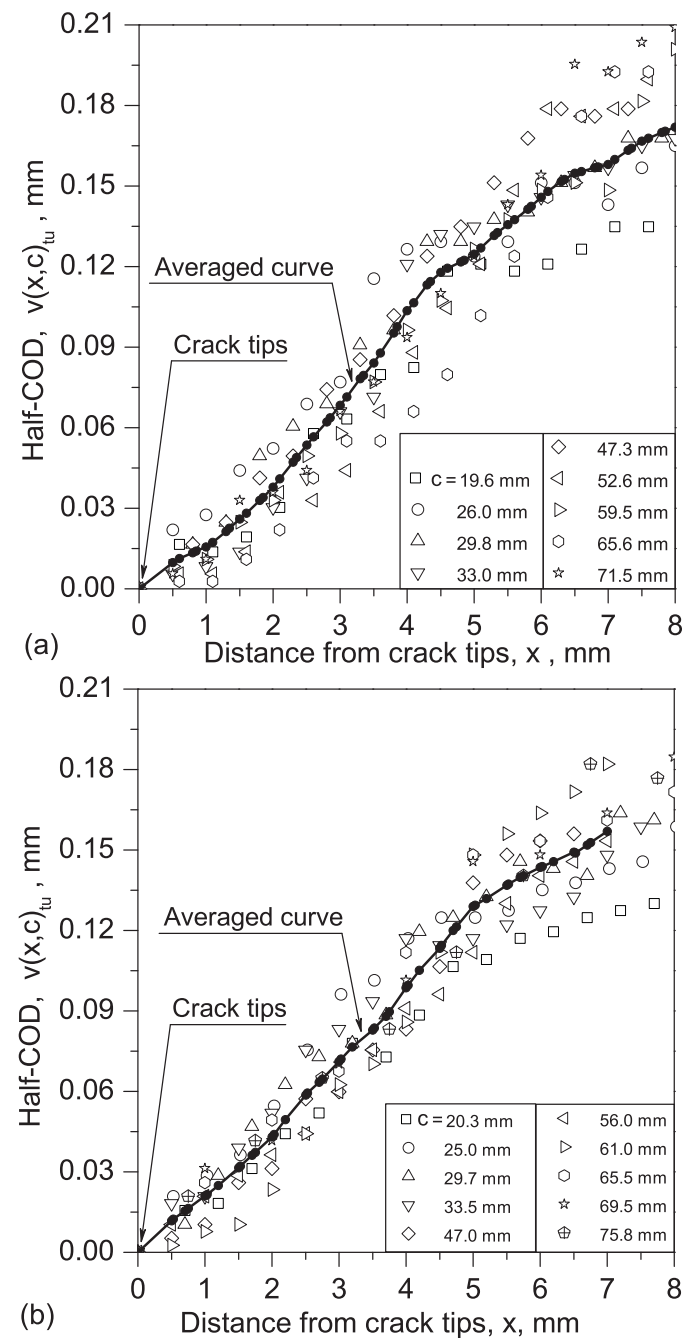

Fig. (14). Crack opening displacements measured near the tips of tear cracks of different lengths in two identical MM(T)-1.0-2.0 (LT direction) specimens during their unloading from the instant $t$ designating the termination of loading to the instant $u$ designating the state of complete unloading.

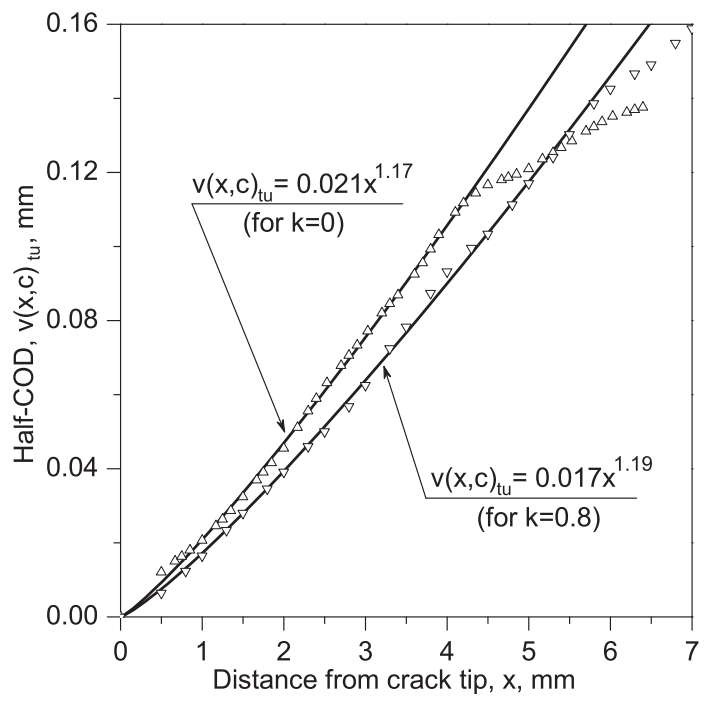

Fig. (15). Comparison of crack-tip dominated regions of the tear crack profiles in uniaxially-loaded $\mathrm{MM}(\mathrm{T})-1.0-2.0$ and biaxiallyloaded MM(T-TC)-1.0-2.0 specimens (both tested along the LT direction) used to calculate the local parameters of the crack profile. 


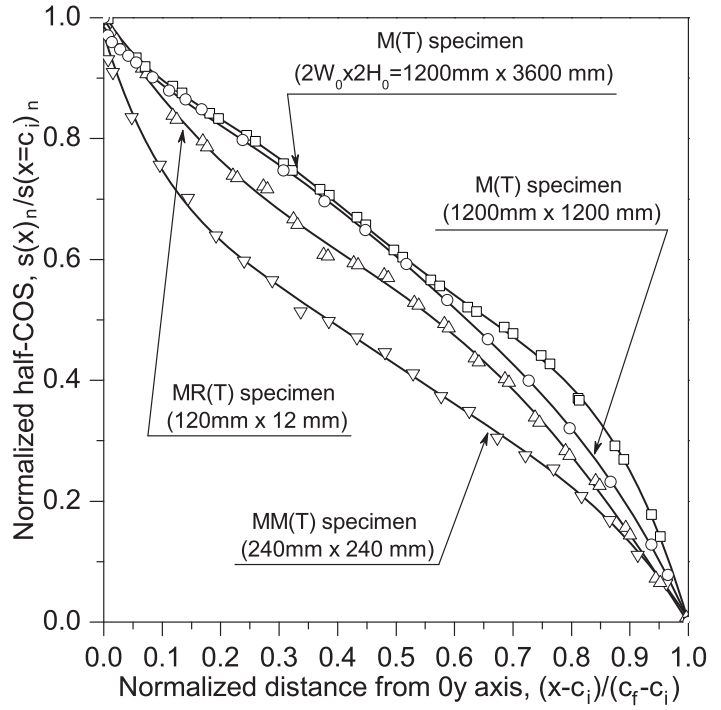

Fig. (16). Normalised presentation of one-quarter crack profiles obtained for fully-fractured specimens of different geometries and sizes that were tested under uniaxial tension in the LT direction.
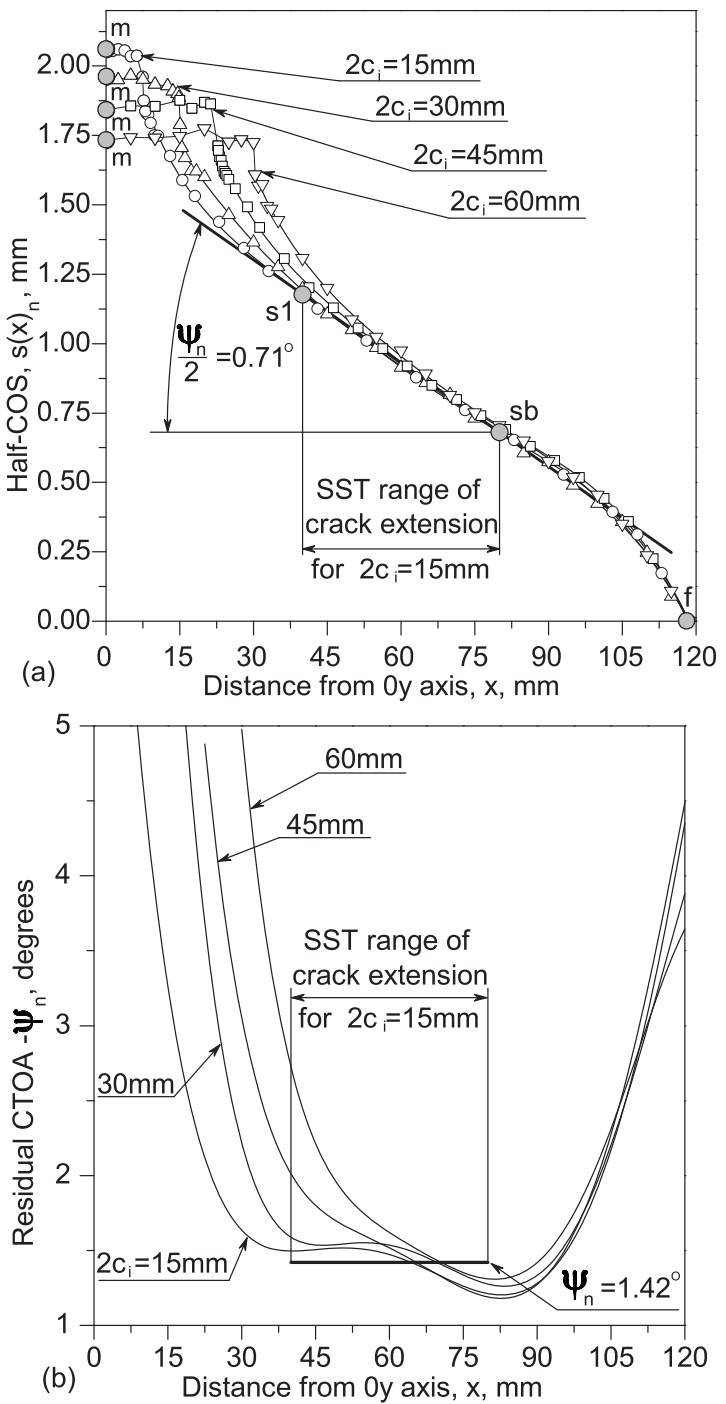

Fig. (17). Through-life fracture curves expressed in terms of the residual COS (a) and residual CTOA (b) for MM(T)-1.0-2.0 (TL orientation) specimens containing initial notches of different lengths $2 \mathrm{c}_{\mathrm{i}}$.

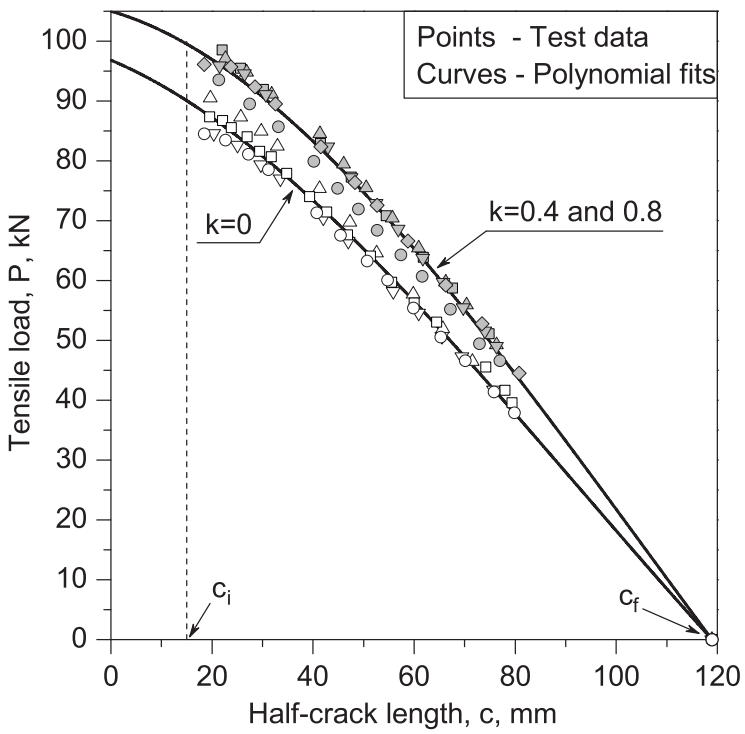

Fig. (18). Experimental diagrams for $\mathrm{MM}(\mathrm{T})-1.0-2.0$ (LT direction) specimens tested in uniaxial tension and MM(T-TC)-1.0-2.0 (LT direction) specimens tested in biaxial tension.

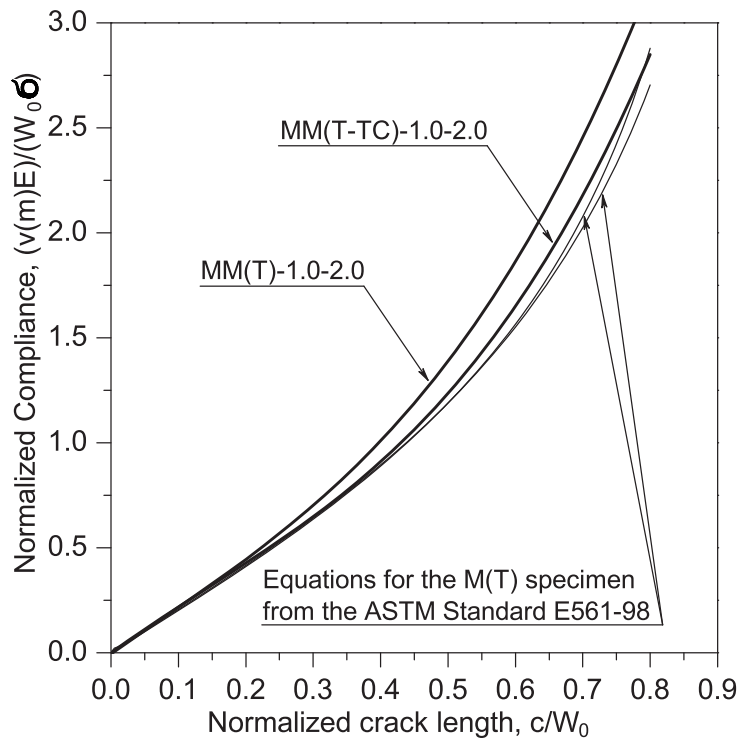

Fig. (19). Comparison of experimentally determined and calculated compliance curves for cracks in two identical MM(T)-1.0-2.0 specimens (LT direction). These curves were established on the assumption that during unloading-reloading cycles the test record can be represented by a straight line passing through the characteristic points $t$ and $u$ (see Fig. 6). It means that the hysteresis behaviour observed was completely neglected. They are shown together with the averaged compliance curves for the MM(T-TC)-1.0-2.0 (LT direction) specimens tested at $k=0.4$ and 0.8 .

It should be emphasised that the current FFS procedures typically employ the following presumption: the yield strength $\sigma_{\mathrm{Y}}$ and the ultimate tensile strength $\sigma_{\mathrm{UTS}}$, being the averaged net-section stresses, can be directly related to the critical parameters of local (near crack-tip) stress fields like $K_{\mathrm{c}}$ or $J_{\mathrm{c}}$. As to the conceptual inconsistency of this statement, it is sufficient to say that the often used characteristics of tensile strength and fracture resistance all are size- and constraintdependent quantities. Therefore, the length scale presented by equation (7) cannot be coupled in a simple and unified manner 


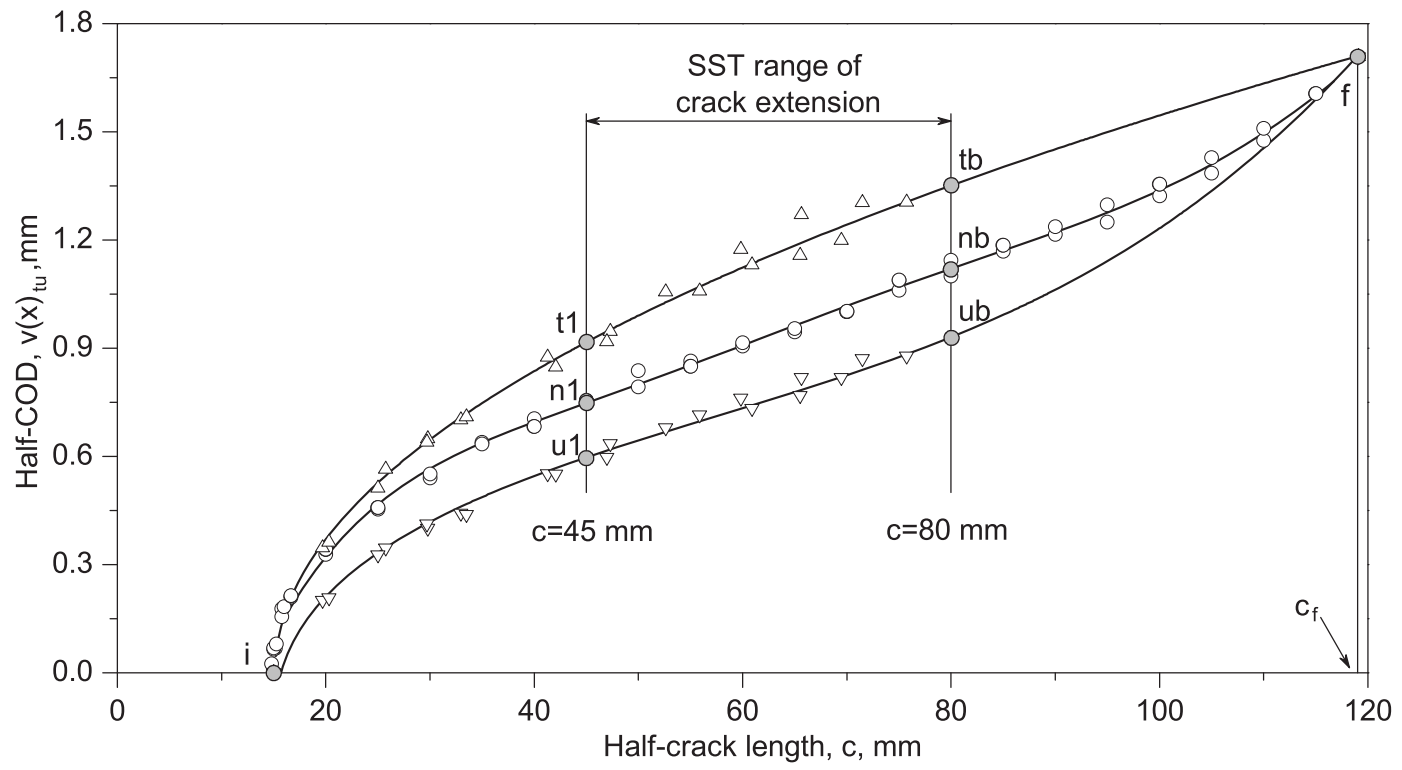

Fig. (20). Through-life fracture curves for two $\mathrm{MM}(\mathrm{T})-1.0-2.0$ (LT direction) specimens with an identical original notch of length $2 c_{\mathrm{i}}=30$ $\mathrm{mm}$. Both specimens were tested with the unloading-reloading cycles.

with the parameters characterising the global fracture behaviour of stationary and extending tear cracks. In other words, the characteristics like $K_{\mathrm{c}}, \sigma_{0}$, and $r_{0}$ cannot be used in a consistent FFS procedure as interrelated physical properties of a substance.

Our experimental results call into question the very meaning of the current approach to the characterisation of ductile tearing resistance in terms of the CTOA- $\psi_{\mathrm{c}}$ parameter. It is seen from the data presented in Figs. (25b), (26), and (27b) that the angle $\psi$, as defined in standard test methods $[21,22]$, usually does not reach a distinct constant level $\psi_{\mathrm{c}}$. This is also the case with the fracture resistance characterization using both procedures based on equations (1) and (2). The $\delta$-R curves in Fig. (27a) can be treated as close prototypes of R curves expressed in terms of the CTOA$\delta_{5}$ values.

It is supposed that the valid criterion of SST crack growth should be reasonably independent of, at least, the PD geometry and size, as well as the geometry and size of the initial imperfection. The criterion values $\psi_{\mathrm{c}}, \psi_{\mathrm{n}}$, and $\psi_{\mathrm{du}}$ all depend on these geometric variables and also on the boundary restraints [53]. Besides, they are influenced by changes in the preloading history (Fig. 25a), by load biaxiality (Fig. 23a), and only slightly by plastic anisotropy (Table 2 ). In comparison with the angle $\psi_{\mathrm{c}}$, the novel characteristics $\psi_{\mathrm{n}}$, and $\psi_{\mathrm{du}}$ are more consistent and reproducible quantities. Obviously, the large scatter in CTOA- $\psi_{\mathrm{c}}$ (Fig. 25b) does not allow us to distinguish the small differences in the tearing resistance due

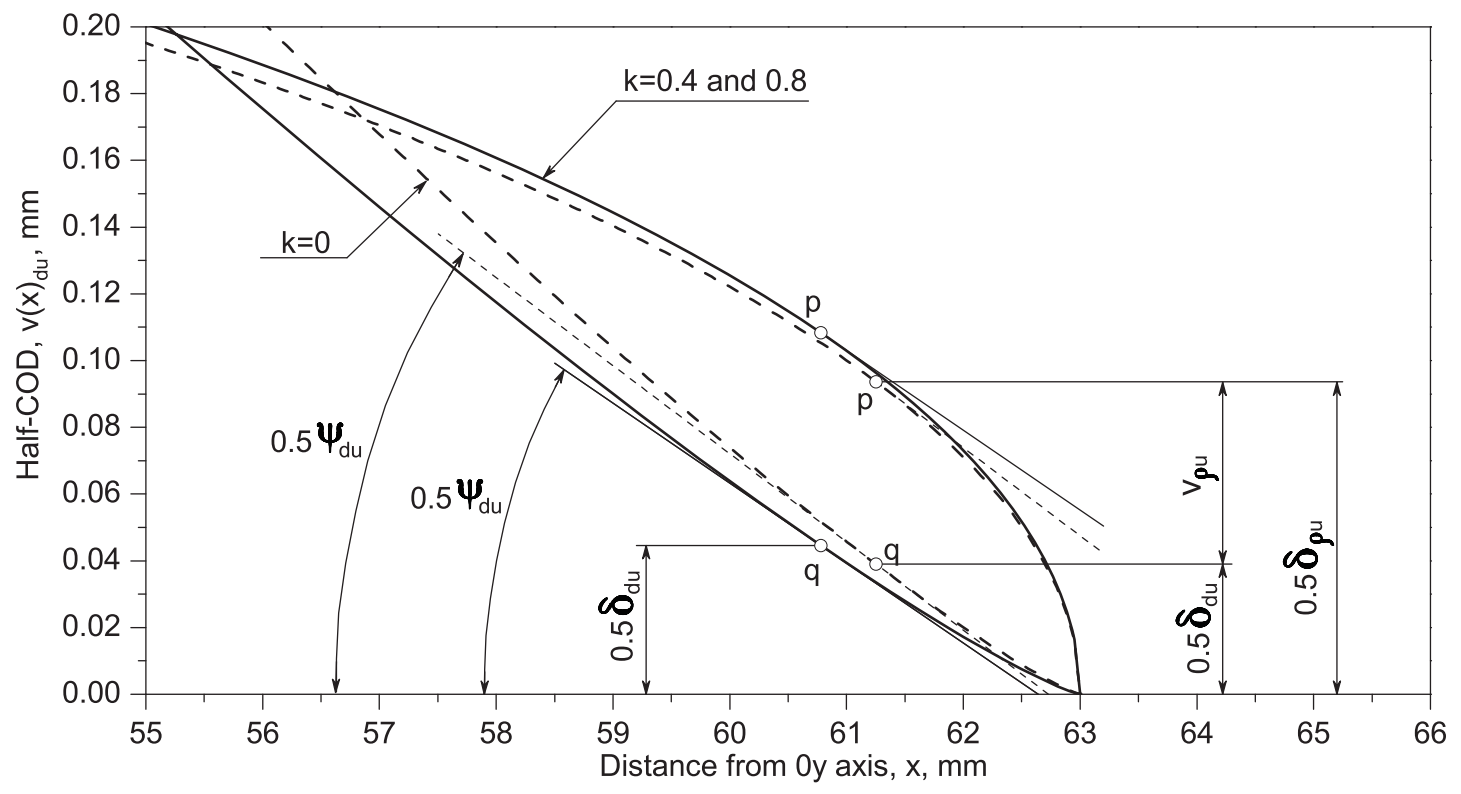

Fig. (21). Comparison of near crack-tip dominated regions for MM(T)-1.0-2.0 and MM(T-TC)-1.0-2.0 specimens (both tested along the LT direction), when the length of tear cracks at the onset of unloading was equal. 

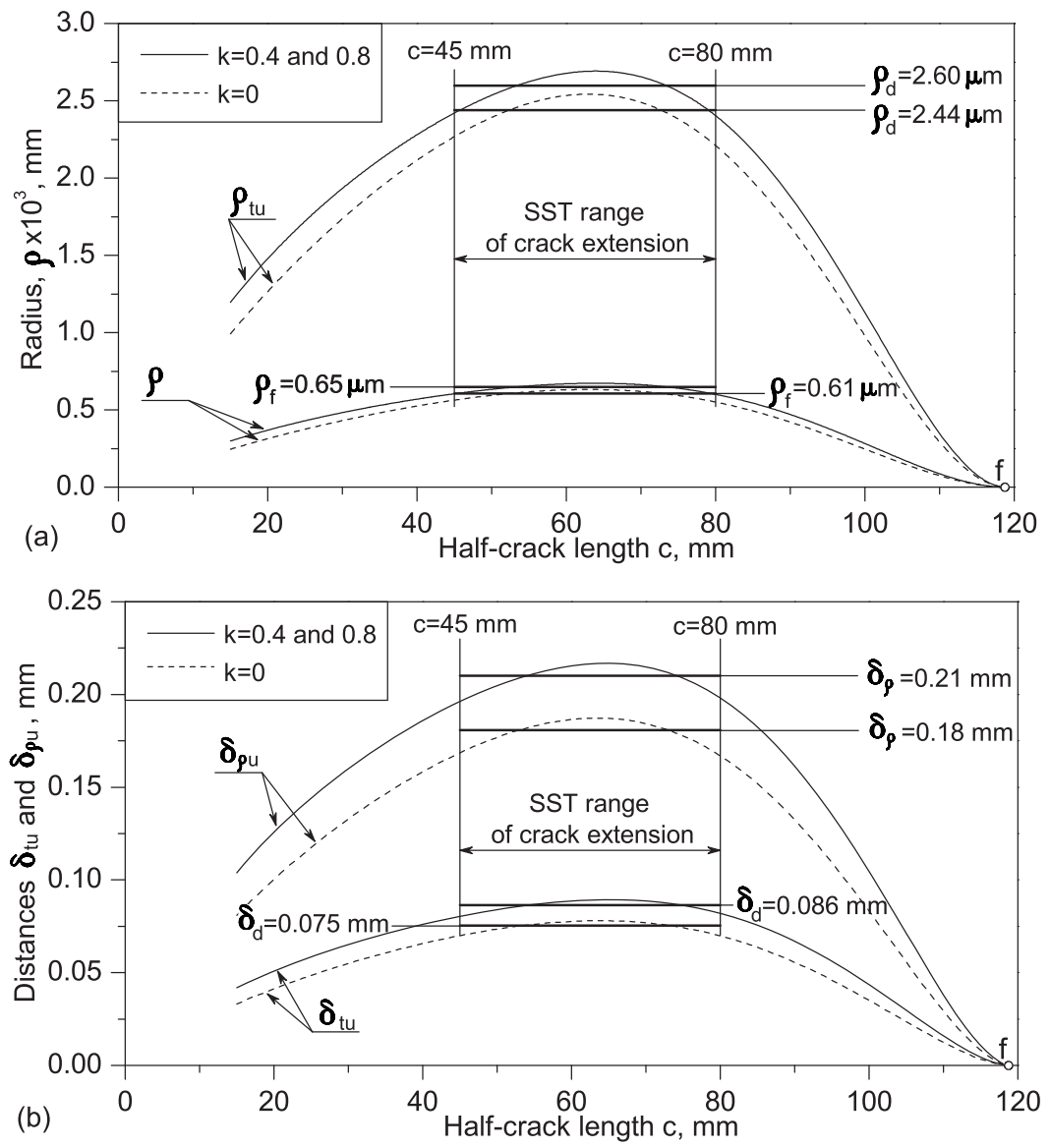

Fig. (22). Variations in the crack-tip radii $\rho$ and $\rho_{\mathrm{tu}}$ in $\mathrm{MM}(\mathrm{T})-1.0-2.0$ and $\mathrm{MM}(\mathrm{T}-\mathrm{TC})-1.0-2.0$ specimens (both tested along the LT direction) (a) and the related variations in the characteristic distances between the imaginary crack tips (b).

to relatively weak effects of plastic anisotropy, loading history, and load biaxiality.

It seems likely that the CTOD- $\delta_{5}$ and CTOA- $\psi_{c}$ concepts need to be modified or replaced by a necessarily more pragmatic approach to assessing the ductile tearing resistance. From this standpoint their combined use with displacement-based characterisations of tearing developed in the framework of the UM is very promising. Being directly connected with parameters of the global fracture behaviour, the angles $\psi_{n}$ and $\psi_{d u}$ in comparison with the CTOA- $\psi_{c}$ are easy to obtain in an unadulterated form. This advantage comes into a particular prominence in tests of brittle materials, when the $\psi_{c}$ angles are comparatively small (fractions of a degree). Clearly, a sufficiently general engineering procedure must allow quantification of the fracture toughness for brittle and ductile materials in a simple and unified manner. The special convenience of the UM concept is that the angles $\psi_{n}$ and $\psi_{d u}$ can be easily incorporated into the energy-based analysis of SST using the test records with loading-unloading cycles shown in Fig. (6).

\section{GENERAL REMARKS}

Although this study is still in progress, the experimental results appear to be sufficiently significant to make the following general conclusions. The constraint-dependent formation and extension of an NFC is governed by the interplay of some set of linear and angular geometric variables. This irreversible process occurs in an orderly sequence and a temporally discontinuous manner. The UM relationships between length scales and specific angles of the SST crack growth can be readily incorporated in an engineering procedure for assessing the plane stress tearing resistance. It is precisely this line of research that can lead us to establishing a simple TL, i.e., correlations between local parameters of a moving crack-tip in laboratory-sized specimens and parameters characterising the fracture behaviour of large-scale components.

The overall results of our work suggest that there are a number of basic distinctions of the UM from the currently used concept of plane stress tearing. The most important among them are as follows:

1. A through crack is presented in fracture analysis by simple model equations describing the difference in distances between the crack borders in a loaded and completely unloaded specimen. It means that the deformed configuration of a tear crack profile serves as the reference state.

2. The tear crack growth is treated in fracture analysis as a step-wise extension of a two-tier model of the crack border along the mutually perpendicular directions.

3. The novel criterion of ductile tearing instability states that the transition from stable to unstable cracking occurs at the instant where the system materialspecimen-loading device attains its minimum capacity 
to absorb permanent strains and structural damage (measured by the $\psi_{\mathrm{n}}$ angle) simultaneously with the attainment of the maximum crack-driving force (measured by the $\psi_{\mathrm{du}}$ angle).

4. The description of the cracking initiated from a small geometric imperfection is in harmony with the description of the crack growth starting from the tips of an elongated stress raiser.

5. The displacement-based characteristics of the cracktip and crack-mouth dominated regions on a centre crack border are related to each other in a clear-cut manner and all of them can be extracted directly from the macroscopic measurements.
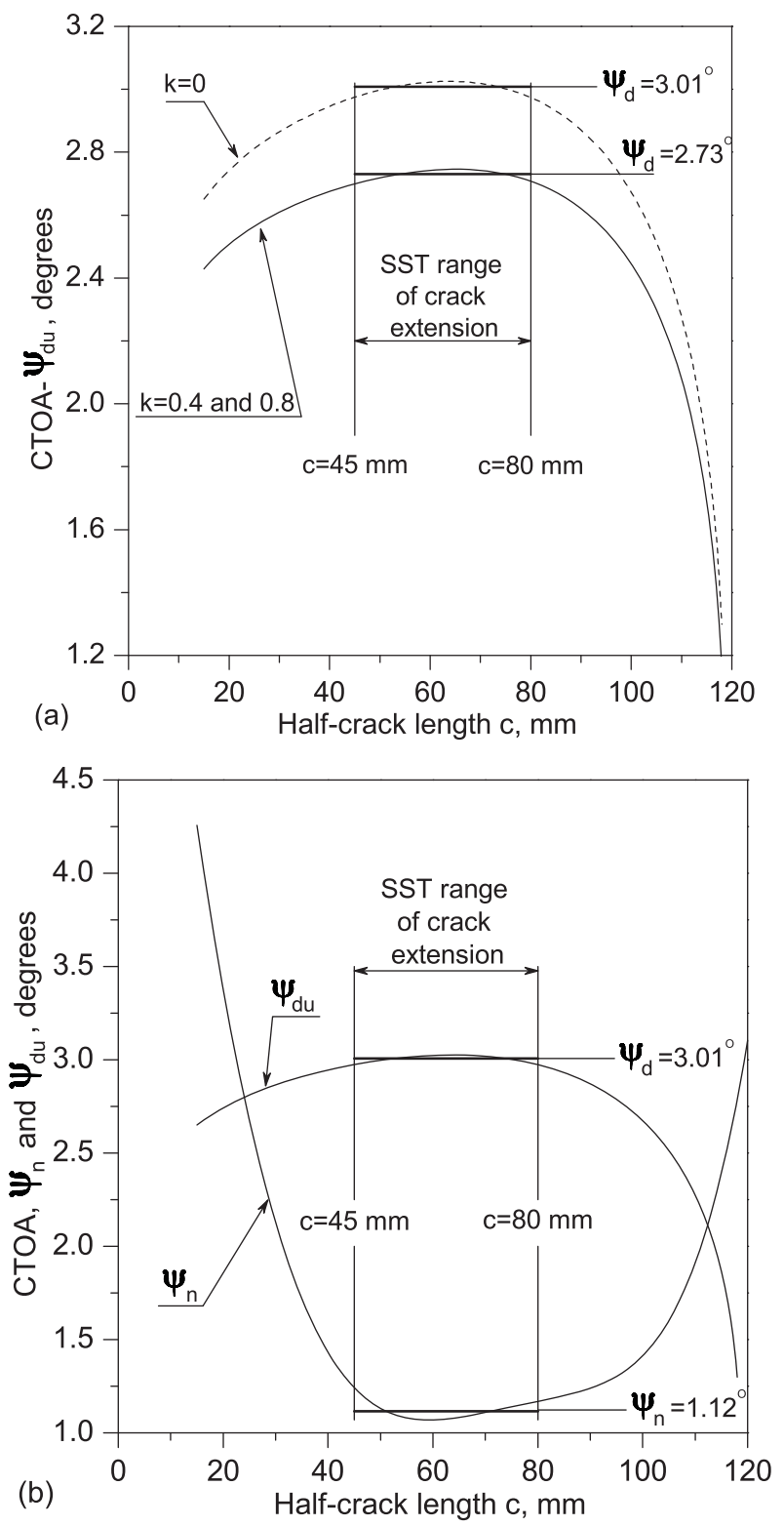

Fig. (23). Variations in the active components of resistance to plane stress tearing under uniaxial and biaxial tension in the $\mathrm{MM}(\mathrm{T})-1.0-2.0$ and $\mathrm{MM}(\mathrm{T}-\mathrm{C})-1.0-2.0$ specimens with an original crack of length $2 c_{\mathrm{i}}=30 \mathrm{~mm}$ (LT direction) both tested with unloading-reloading cycles (a) and a comparison of the active and residual components of the angle $\psi$ for the given $\mathrm{MM}(\mathrm{T})-1.0-2.0$ specimen (b).

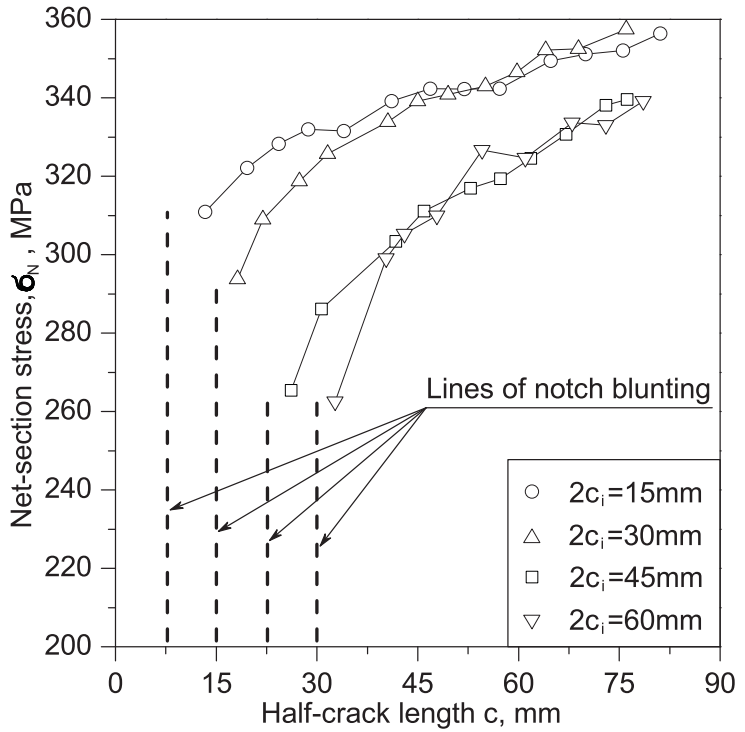

Fig. (24). A set of experimental diagrams for $M M(T)-1.0-2.0$ (TL direction) specimens with original notches of different lengths $2 c_{\mathrm{i}}$. The specimens were tested with the termination of loading (without performing the cycles of unloading-reloading).

6. The length scales reflecting variations in the geometry of the crack-tip dominated region are introduced in fracture analysis without an a priori assumption of the existence of material constants like $K_{\mathrm{c}}, J_{\mathrm{c}}$, and $\sigma_{0}$. That is why they fit naturally into the stress- and energy-based descriptions of the SST crack growth.

7. Attention is redirected from analysing stress-strain fields in the vicinity of an ideal crack tip to describing the behaviour of the entire crack border where the effects of buckling, plasticity, structural damage, and interaction between the inner and outer boundaries all are readily accounted for from test records. As a result, our semi-analytic approach allows prediction of fracture instability events without numerical simulations of the near crack-tip stress and strain fields.

8. The stable crack growth in thin sheets of brittle and ductile materials can be studied using fracture toughness characteristics with the same physical meaning;

9. The displacement-based characteristics of SST resistance can be easily separated into constituents suitable for predicting the mechanical behaviour of large-scale components from data collected on simple specimens of relatively small size.

These distinctive features make it reasonable to assume that the UM can provide a sound framework for the future development of a general procedure for a unified assessment of stable crack growth in damage-tolerant structural elements made from thin sheets of brittle and ductile materials subjected to tensile and/or compressive loading. However, due to a developing nature of the UM, there are several uncertainties over the end result of our explorations.

The main ones are the small number of tests on large-scale specimens and the lack of complete understanding how the SST characteristics can be correlated with the 

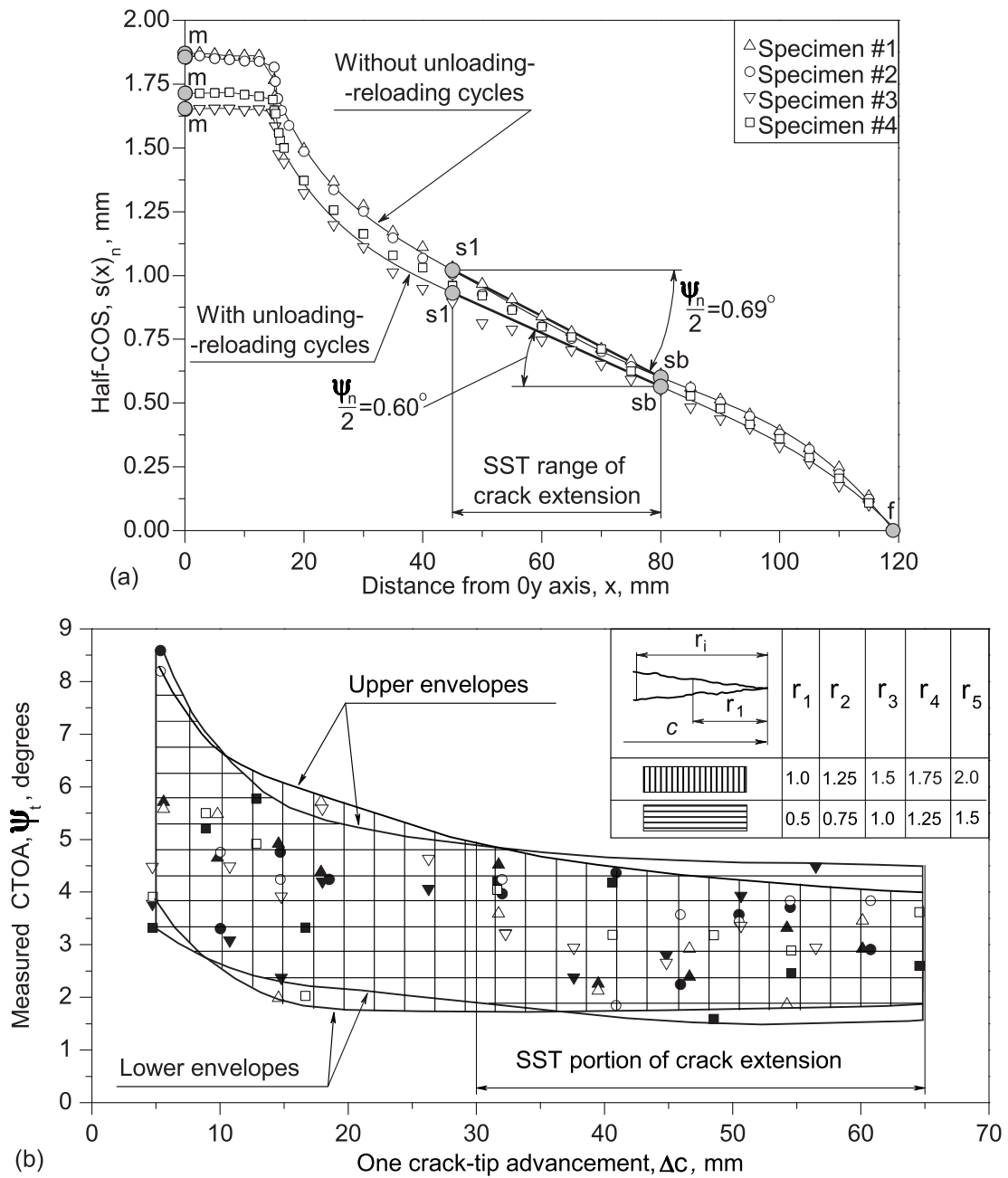

Fig. (25). Effects of introducing the unloading-reloading cycles on the COS-s $(\mathrm{x})_{\mathrm{n}}$ in four MM(T)-1.0-2.0 (LT direction) specimens with an identical stress raiser of length $2 \mathrm{c}_{\mathrm{i}}=30 \mathrm{~mm}$ (a) and the CTOA- $\psi_{\mathrm{t}}(\mathbf{b})$ determined for these specimens using two versions of the data processing procedure developed in [52].

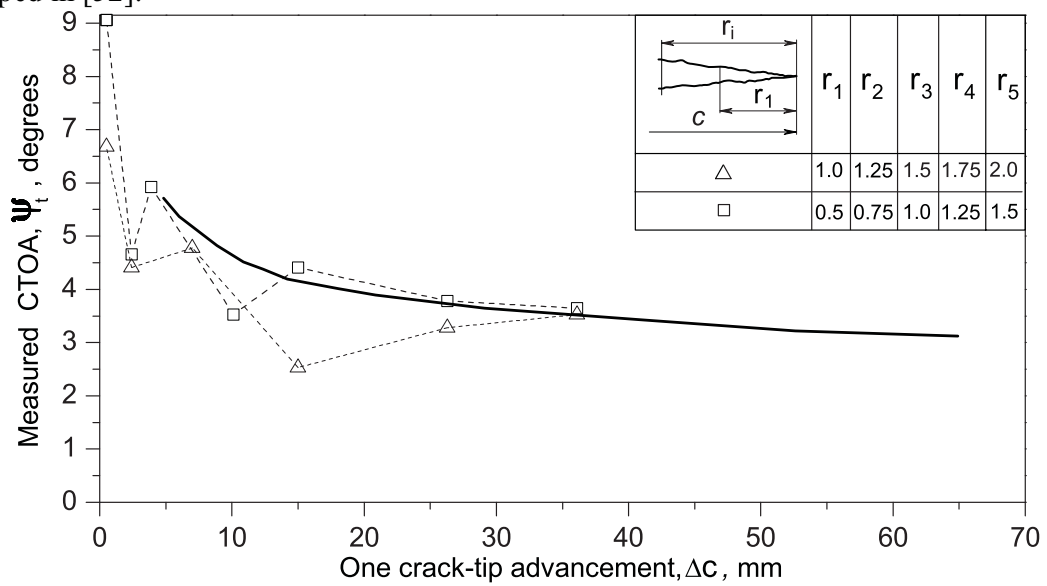

Fig. (26). Comparison of CTOA- $\psi$ angles (points) determined for the large-scale specimen $\mathrm{M}(\mathrm{T})-1.0-10.0$ using two versions of the data processing procedure developed in [52] and the mean curve obtained by averaging the data in Fig. (25b).

micromechanical parameters of the moving tips of a slant crack. The limited amount of experimental data generated by this investigation for large-size specimens (Table 2) cannot offer a sufficient basis for an extensive discussion of the size-scale effects. In addition, we deal only with a purely mechanistic approach, not taking into account the microstructural and physical aspects of the fracture process. It is clear that the results obtained in the framework of such an approach cannot be applied blindly in unexplored areas, i.e., for predicting fracture behaviour of other geometries and materials. 

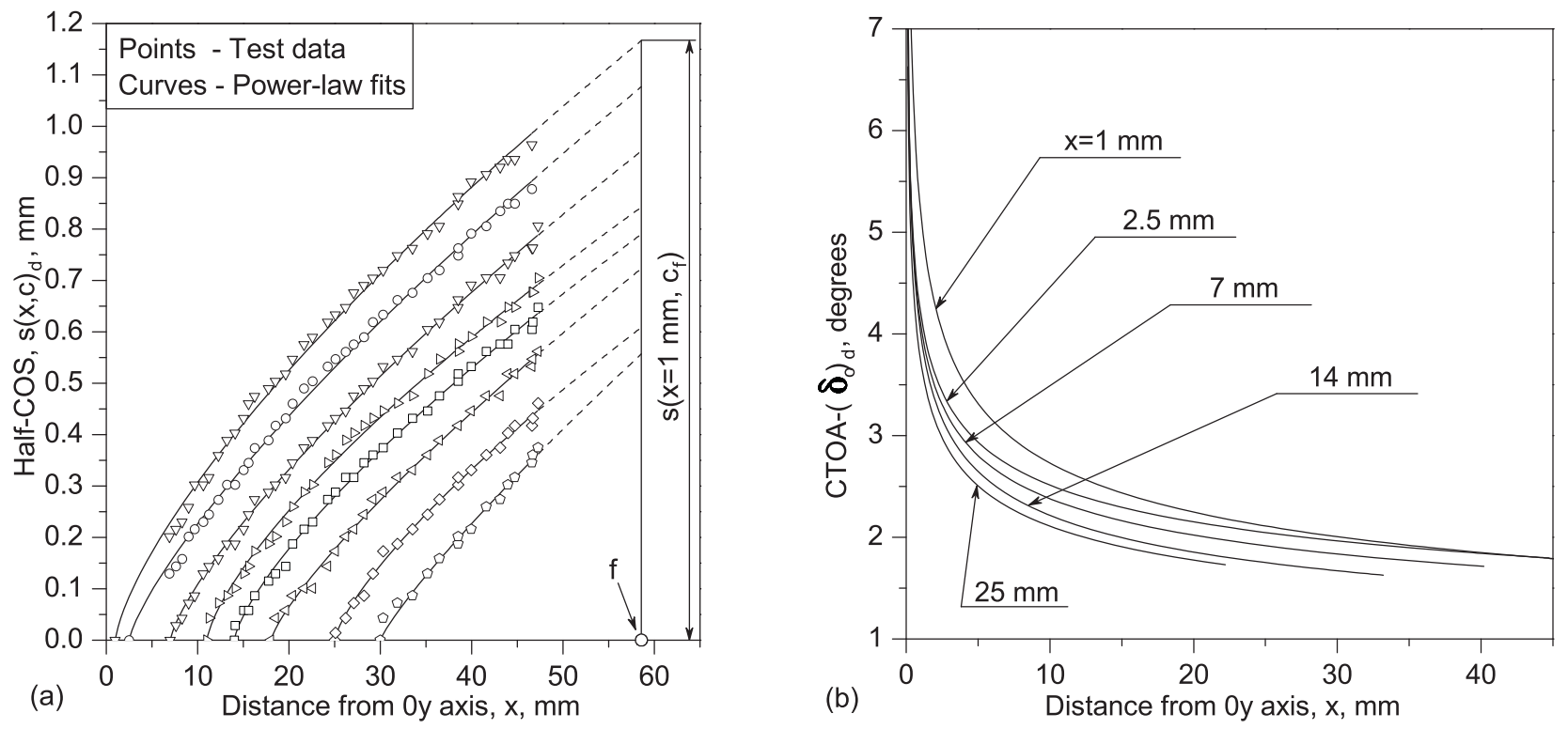

Fig. (27). Variation in the spacing between fixed points on the profile of a continuously moving crack in an MR(T)-1.0-1.0 specimen (a) and the related CTOA- $\left(\delta_{0}\right)_{\mathrm{d}}$ curves $(\mathbf{b})$ determined from equation (2) on a reasonable assumption that $\delta_{0}=2 s(x, c) \approx \delta_{5}$. The difference between the $\delta_{0}$ and $\delta_{5}$ values tends to become negligible as the crack elongates under continuous tearing.

At present, the UM may be thought of mainly as a complementary approach to the commonly used fracture criteria and test methods. Our experimental findings and some theoretical novelties derived from them can already be used for further refining of the engineering concept discussed in [20-22]. At the same time, the UM has a distinct potential to be treated as an alternative semianalytic concept of ductile tearing. This assertion emerges from the aforementioned distinctions between the UM and the conventional methodology of fracture investigation. The experimental results of this study demonstrate that a universal law governing the SST crack growth has not been found yet. However, there are good reasons to believe that in the long run it will be possible to adequately cover at least the main experimental facts using the UM analysis.

\section{ABBREVIATIONS}
CTOA
$=$ Crack Tip Opening Angle
$\mathrm{CTOA}-\psi_{\mathrm{c}}=$ Critical value of the CTOA
$\mathrm{CTOA}-\psi_{\mathrm{n}}=$ Residual component of the CTOA
$\mathrm{CTOA}-\psi_{\mathrm{du}}=$ Active component of the CTOA
CTOD $=$ Crack Tip Opening Displacement
CTOD- $\delta_{5}=$ CTOD defined for a gauge length of $5 \mathrm{~mm}$

Table 2. Characteristics of SST Crack Growth in Thin-Sheet Aluminium Alloy D16AT

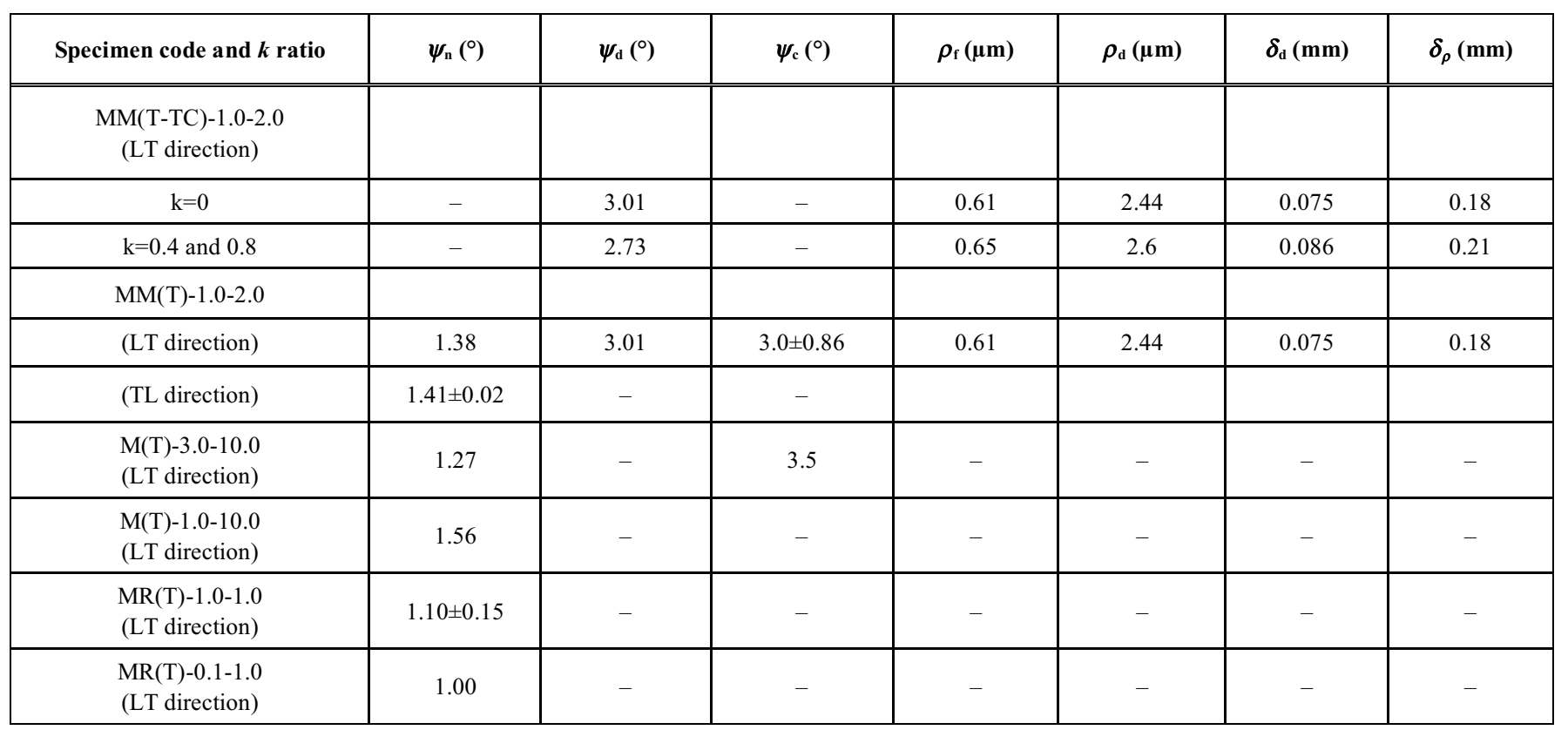




\begin{tabular}{|c|c|c|}
\hline CTOD- $\delta_{\mathrm{d}}$ & $=$ & $\begin{array}{l}\text { CTOD defined for the crack-tip } \\
\text { dominated region }\end{array}$ \\
\hline CTOD- $\delta_{\rho}$ & $=$ & $\begin{array}{l}\text { CTOD defined for an ideal crack } \\
\text { represented by an open elliptic hole }\end{array}$ \\
\hline COD & $=$ & Crack Opening Displacement \\
\hline $\mathrm{COD}-v_{\mathrm{du}}$ & $=$ & $\begin{array}{l}\text { COD defined as the difference between } \\
\text { values of the COS for loaded and fully } \\
\text { unloaded specimen }\end{array}$ \\
\hline CTOS- $\delta$ & $=$ & Crack Tip Opening Spacing \\
\hline CMOS- $2 s(\mathrm{~m})$ & $=$ & Crack Mouth Opening Spacing \\
\hline COS-2s & $=$ & Crack Opening Spacing \\
\hline BSE & $=$ & Basic Structural Element \\
\hline $\mathrm{PD}$ & $=$ & Problem Domain \\
\hline $\mathrm{M}(\mathrm{T})$ & $=$ & Middle-cracked Tension specimen \\
\hline $\mathrm{MM}(\mathrm{T})$ & $=$ & Modified M(T) specimen \\
\hline $\mathrm{MM}(\mathrm{T}-\mathrm{TC})$ & $=$ & $\begin{array}{l}\text { Modified Middle-cracked cruciform } \\
\text { specimen }\end{array}$ \\
\hline $\operatorname{MR}(\mathrm{T})$ & $=$ & $\begin{array}{l}\mathrm{M}(\mathrm{T}) \text { specimen with an open circular } \\
\text { hole of the centre location }\end{array}$ \\
\hline SST & $=$ & Steady State Tearing \\
\hline TET & $=$ & Tail End Tearing \\
\hline NFC & $=$ & Naturally Forming Crack \\
\hline ADZ & $=$ & Active Damage Zone \\
\hline TL & $=$ & Transferring Law \\
\hline
\end{tabular}

\section{REFERENCES}

[1] G. S. Pisarenko and V. P. Naumenko, "Experimental Methods of the Materials Fracture Mechanics", Soviet Material Science, vol. 18, No. 2, pp. 110-120, 1982.

[2] G. S. Pisarenko, V. P. Naumenko and E. E. Onishchenko, "Elasto-Plastic Mode I Fracture of Thin-Sheet Steel under Biaxial Loading" [in Russian], Preprint of the Institute for Problems of Strength of the Ac. Sci. of Ukr. SSR, Kiev, 64 pp., 1983.

[3] G. S. Pisarenko, V. P. Naumenko, O. V. Mitchenko and G. S. Volkov, "Experimental determination of the value of $K_{\mathrm{I}}$ in compression of a plate along the crack line", Strength of Materials, vol. 16, No. 11, pp. 1497-1505, 1984.

[4] G. S. Pisarenko, V. P. Naumenko and V. M. Stepkov, "Method and results for experimental investigation into fracture kinetics and resistance to crack growth in brittle materials", Strength of Materials, vol. 16, No. 12, pp. 1694-1702, 1984.

[5] V. P. Naumenko and O. V. Mitchenko, "Brittle fracture of a plate with a hole in compression", Strength of Materials, vol. 17, No. 7, pp. 890-900, 1985.

[6] G. S. Pisarenko, V. P. Naumenko and N. I. Nedelchev, "Brittle material crack resistance characteristic: what is it", Strength of Materials, vol. 17, No. 11, pp. 1497-1507, 1985.

[7] G. S. Pisarenko, V. P. Naumenko and V. M. Stepkov, "Brittle fracture in uniaxial and biaxial loading" [in Russian], Strength of Materials and Structural Components under a Complex Stress State, Kiev, Naukova Dumka, pp. 196-201, 1986.

[8] V. P. Naumenko, "Brittle Fracture and Strength of Materials under Compression and Tension" [in Russian], Preprint, the Institute for Problems of Strength of the Ac. Sci. of Ukr. SSR, Kiev, 1987, 38p.

[9] V. P. Naumenko, "Nucleation and growth of a macrocrack in tension and compression", Soviet Material Science, vol. 27, No. 5, pp. 484-488, 1991.
[10] V. P. Naumenko and O. V. Mitchenko, "Deformation parameters of Mode I fracture in tension and compression", Strength of Materials, vol. 24, No. 8, pp. 1072-1080, 1991.

[11] V. P. Naumenko, "Modeling of brittle fracture in tension and compression," in RILEM/ESIS Conference on Fracture Processes in Concrete, Rock and Ceramics, J. G. M. van Mier, J. G. Rots and A. Bakker, Eds. London: E \& F.N. Spon, pp. 183-192, 1991.

[12] V. P. Naumenko and S. I. Sopenko, "The test method for crack growth resistance and elasticity characteristics evaluation for brittle nonmetallic materials," in RILEM/ESIS Conference on Fracture Processes in Concrete, Rock and Ceramics, J. G. M. van Mier, J. G. Rots and A. Bakker, Eds. London: E \& F.N. Spon, pp. 183-192, 1991.

[13] V. P. Naumenko and V. A. Rakovsky, "Crack growth onset in biaxially loded elasto-plastic plates," in ESIS/EGF9 Conference on Defect Assessment in Components-Fundamentals and Application, Berlin, pp. 363-377, 1991.

[14] V. P. Naumenko, "Griffith problem and Mode I fracture in tension and compression", Material Science, vol. 29, No. 4, pp. 396-405, 1993.

[15] V. P. Naumenko and O. Kolednik, "Load biaxiality effect on the fracture resistance of thin steel sheets," in 10th European Conference on Fracture (ECF 10) Structural Integrity: Experiments, Models and Application, Berlin, vol. 2, pp. 911-921, 1994.

[16] V. P. Naumenko and G. S. Volkov, "Assessment of plane stress tearing in terms of various crack-driving parameters", Fatigue and Fracture Mechanics, ASTM STP1461, S. R. Daniewicz, J. C. Newman, Jr., and K.-H. Schwalbe, Eds. West Conshohocken, PA: ASTM International, vol. 34, 20 pages, 2003.

[17] V. P. Naumenko, Yu. D. Skrypnyk and N. I. Nedelchev "Constraintdependent fracture toughness of glass and PMMA," in $11^{\text {th }}$ International Conference on Fracture, Turin, 6 pages, 2005.

[18] J. Eftis, D. L. Jones, and H. Liebowitz, "Load biaxility and fracture: synthesis and summary", Engineering Fracture Mechanics, vol. 36, No. 4, 537-574, 1990.

[19] FITNET, European Fitness-For-Service Network, Final report for Work Package 2 "State-of-the-Art and Strategy", October 2003.

[20] The Special Issue on Fundamentals and Applications of the Crack Tip Opening Angle (CTOA), Engineering Fracture Mechanics, vol. 70, No. 3-4, 371-577, 2003.

[21] Working document ISO/TC 164/SC 4 N413.3, Metallic Materials Method of Test for the Determination of Resistance to Stable Crack Extension Using Specimens of Low Constraint, 2004.

[22] ASTM Standard. Test Method for Determination of Resistance to Stable Crack Extension under Low-Constraint Conditions (E2472$06)$.

[23] Y. J. Chao, "On a single parameter controlled fracture of solids under plane stress conditions", International Journal of Fracture, vol. 58, pp. 104-110, 1991.

[24] SINTAP. Structural integrity assessment procedure. EU-Project BE 95-1462:-Final Report.- Brite Euram Programme, Brussels, 1999.

[25] M. Schödel and U. Zebst, "Application of the European flaw assessment procedure SINTAP to thin wall structures", Engineering Fracture Mechanics, vol. 71, pp. 1035-1058, 2004.

[26] L. Xia, C. F. Shih and J. W. Hutchinson, "A computational approach to ductile crack growth under large scale yielding conditions", Journal of Mechanics and Physics of Solids, vol. 43, No. 3, pp. 389, 1995.

[27] V. P. Naumenko, O. Kolednik, N. P. O’Dowd, G. S. Volkov and A. I. Semenets, "Transferability of plane-stress R-curves: Effect of specimen size and crack length" in ECF 12 Fracture from Defects, M. W. Brown, E. R. de los Rois, and K J. Miller, Eds., vol. 2, pp. 631636, 1998.

[28] V. P. Naumenko, O. Kolednik, N. P. O'Dowd and G. S. Volkov, "Effect of constraint on resistance to stable crack growth in thin aluminium plates", in Intern. Conference "Life Assessment and Management for Structural Components", Kiev, Ukraine, vol. I, pp. 299-304, 2000.

[29] V. P. Naumenko, G. S. Volkov and A. G. Atkins, "Comparison studies of R-curves for fatigue and tear cracks in a thin aluminum plate", in ECF 13 Fracture Mechanics: Applications and Challenges, San Sebastian, Spain, 2000, Online, reference No. 1A.34, 8 pages.

[30] J. C. Newman, Jr., D. S. Dawicke and C. A. Bigelow, "Finite-element analysis and measurement of CTOA during stable tearing in a thinsheet aluminum alloy", in International Workshop on Structural Integrity of Aging Airplanes, Atlanta, pp. 167-186, 1992. 
[31] V. P. Naumenko, G. S. Volkov, and A. G. Atkins, "Initiation and propagation of ductile tearing: A search for biaxial fracture criterion", in the Sixth International Conference on Biaxial/Multiaxial Fatigue and Fracture, M. M. de Freitas, Ed. Lisboa, pp. 975-982, 2001.

[32] V. P. Naumenko and Yu. D. Skrypnyk, "Sensitivity of crack nucleation parameters to the geometric imperfection", in the ECF16 Fracture of Nano and Engineering Materials and Structures, E. E. Gdoutos, Ed. Alexandropoulos, Greece, 2006.

[33] V. P. Naumenko, S. V. Lenzion and Yu. D. Skrypnyk, "Ductile tearing in narrow and wide strips of thin-sheet aluminium alloy", in the ESIA8 Throughlife Management of Structures and Components, Manchester, UK, 10 pages, 2006.

[34] W. R. Lloyd, "Microtopography for ductile fracture process characterization. Part 1: Theory and methodology", Engineering Fracture Mechanics, vol. 70, No. 3-4, 387-401, 2003.

[35] J. C. Newman, Jr. "An elastic-plastic finite element analysis of crack initiation, stable crack growth, and instability", in Fracture Mechanics, Philadelphia: ASTM STP 833, pp. 93-117, 1984.

[36] D. S. Dawicke, M. A. Sutton, J. C. Newman, Jr. and C. A. Bigelow, "Measurement and analysis of critical CTOA for thisheet aluminium alloy materials", in $25^{\text {th }}$ National Symposium on Fracture Mechanics, Bethlehem, PA, 1993.

[37] J. B. Bakuskas and J. C. Newman, Jr., "Prediction of stable tearing of 2024-t3 aluminium alloy using the crack-tip opening angle approach", in NASA Technical Memorandum 109023, NASA Langley Research Center, 1993.

[38] K-H. Schwalbe, "Introduction $\delta_{5}$ as an operational definition of the CTOD and its practical use", in Fracture Mechanics, ASTM STP 1256, vol. 26, pp. 763-778, 1995.

[39] G. S. Pisarenko, V. P. Naumenko, and E. E. Onishchenko, “A method for investigating the fracture of sheet materials in biaxial loading”, Strength of Materials, vol. 14, No. 3, pp. 275-282, 1982.

[40] A. Hannon and P.Tiernan, "A review of planar biaxial tensile test systems for sheet metals", Journal of Materials Processing Technology, vol. 198, issues 1-3, 3, pp. 1-13, 2008.

[41] J. J. Kibler and R. Roberts, "The effect of biaxial stresses on fatigue and fracture", Transactions of ASME, Journal of Engineering and Industry, vol. 92, pp. 727-734, 1970.
[42] S. Abou-Sayed, D. Broek, T. P. Forte and R. B. Stonesifer, "Plane stress fracture under biaxial loading", in ICF5 Advances in Fracture Research, D. Francois, Ed. Pergamon Press, vol. 4, pp. 1707-1713, 1981.

[43] S. I. Garwood, T. G. Davey and S. I. Cresswell, "Behavior of A533B under biaxial loading at $+70^{\circ} \mathrm{C}$ ", International Journal of Pressure Vessels and Piping, vol. 36, pp. 199-224, 1989.

[44] C. Dalle Done and H. Döker, "Biaxial load effects on plane stress J$\Delta \mathrm{a}$ and $\delta_{5}-\Delta \mathrm{a}$-curves", in ECF 10 Structural Integrity: Experiments, Models and Applications, Berlin, pp. 891-900, 1994.

[45] V. Boiko, L. N. Karpenko, A. A. Lebedev, N. R. Muzika and O. V. Zagornyak, "Calculation-experimental method for determining crack resistance of material under biaxial loading", Engineering Fracture Mechanics, vol. 64, pp. 203-215, 1999.

[46] D. S. Dawicke, A. S. Gullerud, R. H. Dodds, Jr. and R. W. Hampton, "Residual strength predictions with crack buckling", in The Second Joint NASA/FAA/DoD Conference on Aging Aircraft, C. E. Harris, Ed. NASA CP-208982, pp. 565-574, 1999.

[47] J. Eftis and H. Liebowitz, "On the modified Westergaard equations for certain plane crack problems", International Journal of Fracture Mechanics, vol. 8, No. 4, pp. 383-392, 1972.

[48] J. Schijve, "Some considerations on the Eftis-Liebowitz equation for the COD compliance of a center cracked sheet specimen", Engineering Fracture Mechanics, vol. 55, No. 3, pp. 341-346, 1996.

[49] Y. Lei and B. K. Neale, "The fracture behavior of a center cracked tensile specimen", Fatigue and Fracture of Engineering Materials and Structures, vol. 20, No. 2, pp. 201-216, 1997.

[50] C. E. Turner and O. Kolednik, "A micro and macro approach to the energy dissipation rate model of stable ductile crack growth", Fatigue and Fracture of Engineering Materials and Structures, vol. 17, pp. 1089-1107, 1994.

[51] Li Ming and O. Richmond, "Intrinsic instability and nonuniformity of plastic deformation”, International Journal of Plasticity, vol. 13, No.s 8-9, pp. 765-784, 1997.

[52] J. Heerens and M. Schodel, Engineering Fracture Mechanics, vol. 70, pp. 417-426, 2003.

[53] V. P. Naumenko, S. V. Lenzion and A. G. Atkins, "Global vs. local characterization of crack growth in biaxially stretched plates", in the ESIA9 Engineering Structural Integrity - Research, Development and Application, Beijing, China, 4 pages, 2007. 